\title{
ISOMORPHISM CLASSES FOR HIGHER ORDER TANGENT BUNDLES
}

\author{
ALI SURI
}

\begin{abstract}
The tangent bundle $T^{k} M$ of order $k$, of a smooth Banach manifold $M$ consists of all equivalent classes of curves that agree up to their accelerations of order $k$. In the previous work of the author he proved that $T^{k} M, 1 \leq k \leq \infty$, admits a vector bundle structure on $M$ if and only if $M$ is endowed with a linear connection or equivalently a connection map on $T^{k} M$ is defined. This bundle structure depends heavily on the choice of the connection. In this paper we ask about the extent to which this vector bundle structure remains isomorphic. To this end we define the notion of the $k^{\prime}$ th order differential $T^{k} g: T^{k} M \longrightarrow T^{k} N$ for a given differentiable map $g$ between manifolds $M$ and $N$. As we shall see, $T^{k} g$ becomes a vector bundle morphism if the base manifolds are endowed with $g$-related connections. In particular, replacing a connection with a $g$-related one, where $g: M \longrightarrow M$ is a diffeomorphism, follows invariant vector bundle structures. Finally, using immersions on Hilbert manifolds, convex combination of connection maps and manifold of $C^{r}$ maps we offer three examples to support our theory and reveal its interaction with the known problems such as Sasaki lift of metrics.

Keywords: Banach manifold; Hilbert manifold; Linear connection; Connection map; Related connection, Higher order tangent bundle; Fréchet manifold; lifting of Riemannian metrics.
\end{abstract}

\section{Contents}

1. Introduction

2. Preliminaries

2.1. Lifting of related linear connections to higher order tangent bundles

2.2. $T^{k} M$ as a vector bundle

3. $T^{k} g$ as a vector bundle morphism

4. $T^{\infty} g$ as a vector bundle morphism

5. Applications and examples

6. Appendix

References

\section{INTRODUCTION}

The tangent bundle of order $\mathrm{k}, T^{k} M$, of a smooth manifold $M$ consists of all equivalent classes of curves that agree up to their accelerations of order

2010 Mathematical Subject Classification. Primary 58B20; Secondary 58A05. 
k. This bundle is a natural extension of the notion of the usual tangent bundle (see e.g. 6, 20, 21, 30]). For example in classical mechanics, $T^{k} M$ describes the Generalized Particle Mechanics in the autonomous sense [5].

A vector bundle structure for $T^{k} M, 2 \leq k \leq \infty$, even for $k=2$ is not as evident as in the case of tangent bundle $T M$. In fact it is not always possible to consider $T^{k} M$ as a vector bundle over $M[6,25,26$.

The author in his previous work 26] proved that at the presence of a linear connection on $M$ (or equivalently a connection map on $T^{k} M$ ), $T^{k} M$, $2 \leq k \leq \infty$, admits a vector bundle structure on $M$. Moreover it is shown that every linear connection (or equivalently every Riemannian metric) on $M$, induces a connection map on $T^{k} M$.

As an immediate consequence, our suggested vector bundle structure allows us to solve an old problem of differential geometry formulated by Bianchi and Bompiani [20, namely the problem of prolongation of a Riemannian metric defined on the base manifold $M$ to $T^{k} M$, even for infinite dimensional Hilbertable manifolds [26].

However, as one may have expected, these vector bundle structures depend crucially on the particular connection chosen [6, 7, 25, 26].

In this paper we ask about the extent of this vector bundle dependence. We will show that this dependence is closely related to the notion of related connection maps (or conjugate connections) which will be used for a classification of these vector bundle structures. More precisely we introduce the higher order differential $T^{k} g$ of a smooth map $g: M \longrightarrow N$ between two manifolds $M$ and $N$ and we investigate when $T^{k} g$ is linear on fibres. Linearity of $T_{x}^{k} g, x \in M$, allows us to build a vector bundle morphism $T^{k} g: T^{k} M \longrightarrow T^{k} N$ (see 25 and 7 for the special case $k=2$ ). As a consequence, we show that the vector bundle structure on $T^{k} M$, defined by the aim of a connection map, remains invariant (isomorphic) if it is replaced by a $g$-related connection map, for some diffeomorphism $g: M \longrightarrow M$.

If we take one step further by considering $T^{\infty} M$ and $T^{\infty} N$, as generalized Fréchet vector bundles over $M$ and $N$ respectively ([26]), then proving $T^{\infty} g$ to be a generalized vector bundle morphism becomes much more complicated. More precisely the set of linear maps between Fréchet spaces (the fibre types of $\left(T^{\infty} M, \pi_{M}^{\infty}, M\right)$ and $\left.\left(T^{\infty} N, \pi_{N}^{\infty}, N\right)\right)$ does not remain in the category of Fréchet spaces [12, 22. To get around this difficulty, we employ the projective limit methodology, as in [1, 11, 26, 27] etc., to show that $\left(T^{\infty} g, g\right)$ becomes a generalized vector bundle morphism at the presence of $g$-related connections on $M$ and $N$.

Afterward, as an application, we settle our results to the special case of $f: M \longrightarrow N$, where $f$ is an immersion and $N$ is a Riemannian Hilbert manifold. As a result, this special case tells us that higher order differential of an isometry is again an isometry with respect to the induced Sasaki-type metrics.

Then we check the vector bundle dependence on convex combination of connection maps.

We close this article by addressing, in part, the case of manifold of $C^{r}$ maps between manifolds $M$ and $N$ denoted by $C^{r}(N, M)$. 
Through this paper all the maps and manifolds are assumed to be smooth, but, except in section 4, a lesser degree of differentiability can be assumed.

The readers who are unfamiliar with infinite dimensional manifolds and spaces, can easily replace the model spaces with Euclidean spaces.

Most of the results of this paper are novel even for the case of finite dimensional manifolds.

\section{Preliminaries}

In this section we summarize the necessary preliminary materials that we need for a self contained presentation of our paper and we record our notation.

At various points in this article, we wish to have an explicit formula for higher order differentials of compositions of smooth functions. Hence, we begin with a short description of higher order chain rule. Let $f: \mathbb{I} \subseteq \mathbb{R} \longrightarrow U$ and $g: U \longrightarrow V$ be $k$-times Fréchet differentiable where $U$ and $V$ are open subsets of the Banach spaces $\mathbb{E}$ and $\mathbb{E}^{\prime}$ respectively. Then it is known that $g \circ f$ is $k$-times Fréchet differentiable and

$$
\begin{aligned}
& (g \circ f)^{(k)}(0)= \\
& \sum \frac{k !}{l_{1} ! \ldots l_{i} ! m_{1} ! \ldots m_{k} !} d^{i} g(f(0))\left(f^{\left(l_{1}\right)}(0), \ldots, f^{\left(l_{i}\right)}(0)\right)
\end{aligned}
$$

where the second sum is over all ordered $i$-tuples $\left(l_{1}, \ldots, l_{i}\right)$ of integers $l_{1}, \ldots, l_{i}$ such that $l_{1}+\cdots+l_{i}=k$ and $1 \leq l_{1} \leq \cdots \leq l_{i} \leq k$ with $i$ varying form 1 to $k$. Moreover for any $j \in\{1, \ldots k\}, m_{j}$ is the number of $l_{1}, \ldots, l_{i}$ equal to $j$ ([2], p. 234, [17], p. 359 or [23], p. 262). The coefficient $\frac{k !}{l_{1} ! \ldots l_{i} ! m_{1} ! \ldots m_{k} !}$ will henceforth be denoted by $a_{\left(l_{1}, \ldots, l_{i}\right)}^{k}$.

We proceed with a short description of infinite-dimensional manifolds and their tangent bundles. Let $\mathrm{M}$ be a manifold modeled on the Banach space $\mathbb{E}$. For any $x_{0} \in M$ define

$$
C_{x_{0}}:=\left\{\gamma:(-\epsilon, \epsilon) \longrightarrow M ; \gamma(0)=x_{0} \text { and } \gamma \text { is smooth }\right\} .
$$

As a natural extension of the tangent bundle $T M$ define the following equivalence relation. For $\gamma \in C_{x_{0}}$, set $\gamma^{(1)}(t)=\gamma^{\prime}(t)$ and $\gamma^{(k)}(t)=\gamma^{(k-1)^{\prime}}(t)$ where $k \in \mathbb{N}$ and $k \geq 2$. Two curves $\gamma_{1}, \gamma_{2} \in C_{x_{0}}$ are said to be $k$-equivalent, denoted by $\gamma_{1} \approx_{x_{0}}^{k} \gamma_{2}$, if and only if $\gamma_{1}^{(j)}(0)=\gamma_{2}^{(j)}(0)$ for all $1 \leq j \leq k$. Define $T_{x_{0}}^{k} M:=C_{x_{0}} / \approx_{x_{0}}^{k}$ and the tangent bundle of order $k$ or $k$-osculating bundle of $\mathrm{M}$ to be $T^{k} M:=\bigcup_{x \in M} T_{x}^{k} M$. We denote by $\left[\gamma, x_{0}\right]_{k}$ the representative of the equivalence class containing $\gamma$ and define the canonical projection $\pi_{M}^{k}: T^{k} M \longrightarrow M$ which projects $\left[\gamma, x_{0}\right]_{k}$ onto $x_{0}$.

Let $\mathcal{A}=\left\{\left(U_{\alpha}, \phi_{\alpha}\right)\right\}_{\alpha \in I}$ be a $C^{\infty}$ atlas for $M$. For any $\alpha \in I$ define

$$
\begin{aligned}
\phi_{\alpha}^{k}: \pi_{M}^{k-1}\left(U_{\alpha}\right) & \longrightarrow \phi_{\alpha}\left(U_{\alpha}\right) \times \mathbb{E}^{k} \\
{\left[\gamma, x_{0}\right]_{k} } & \longmapsto\left(\left(\phi_{\alpha} \circ \gamma\right)(0),\left(\phi_{\alpha} \circ \gamma\right)^{\prime}(0), \ldots, \frac{1}{k !}\left(\phi_{\alpha} \circ \gamma\right)^{(k)}(0)\right)
\end{aligned}
$$

Theorem 2.1. The family $\mathcal{A}_{k}=\left\{\left(\pi_{M}^{k}{ }^{-1}\left(U_{\alpha}\right), \phi_{\alpha}^{k}\right)\right\}_{\alpha \in I}$ declares a smooth fibre bundle (not generally a vector bundle) structure for $T^{k} M$ over $M$ [26]. 
Consider the $C^{\infty}\left(T^{k} M\right)$-linear map $\mathbb{J}: \mathfrak{X}\left(T^{k} M\right) \longrightarrow \mathfrak{X}\left(T^{k} M\right)$ s.t. locally on a chart $\left(\phi_{\alpha}^{k}, \pi_{M}^{k-1}\left(U_{\alpha}\right)\right), \mathbb{J}$ is given by

$$
\mathbb{J}_{\alpha}\left(u ; y, \eta_{1}, \ldots, \eta_{k}\right)=\left(u ; 0, y, \eta_{1}, \ldots, \eta_{k-1}\right) .
$$

for any $u:=\left(x, \xi_{1}, \ldots, \xi_{k}\right) \in T^{k} M$ and every $\left(u ; y, \eta_{1}, \ldots, \eta_{k}\right) \in T_{u} T^{k} M$.

Definition 2.2. A connection map on $T^{k} M$ is a vector bundle morphism

$$
K=(\stackrel{1}{K}, \stackrel{2}{K} \ldots, \stackrel{k}{K}): T T^{k} M \longrightarrow\left(\oplus_{i=1}^{k} T M, \oplus_{i=1}^{k} \pi_{M}^{1}, \oplus_{i=1}^{k} M\right)
$$

such that for any $1 \leq a \leq k-1, \stackrel{k}{K} \circ \mathbb{J}^{a}=\stackrel{k-a}{K}$ and $\stackrel{k}{K} \circ \mathbb{J}^{k}=\pi_{M *}^{k}$ [4, 26].

In order to carry out the local structure of a connection map, we state the following lemma according to [26].

Lemma 2.3. Locally on a chart $\left(\pi_{M}^{k-1}\left(U_{\alpha}\right), \phi_{\alpha}^{k}\right)$ there are smooth maps $\stackrel{i}{M_{\alpha}}: U_{\alpha} \times \mathbb{E}^{k} \longrightarrow L(\mathbb{E}, \mathbb{E}), 1 \leq i \leq k$, such that the connection map

$$
K_{\alpha}:=\oplus_{i=1}^{k} \phi_{\alpha}^{1} \circ K \circ T \phi_{\alpha}^{k-1}=\left(\stackrel{1}{K} \alpha, \ldots, \stackrel{k}{K}{ }_{\alpha}\right)
$$

at $\left(u ; y, \eta_{1}, \ldots, \eta_{k}\right) \in T_{u} T^{k} M$ is given by

$$
\begin{aligned}
& \left.K\right|_{U_{\alpha}}\left(u ; y, \eta_{1}, \ldots, \eta_{k}\right)= \\
& \bigoplus_{i=1}^{k}\left(x, \eta_{i}+\stackrel{1}{M_{\alpha}}(u) \eta_{i-1}+\stackrel{2}{M_{\alpha}}(u) \eta_{i-2}+\ldots+\stackrel{i}{M}{ }_{\alpha}(u) y\right) .
\end{aligned}
$$

On the common area of the charts $\left(\pi_{M}^{k}{ }^{-1}\left(U_{\alpha}\right), \phi_{\alpha}^{k}\right)$ and $\left(\pi_{N}^{k-1}\left(U_{\beta}\right), \phi_{\beta}^{k}\right)$, the local components $\stackrel{i}{M}_{\alpha}$ and $\stackrel{i}{M}_{\beta}$ are related via the transformation rule given by remark 2.8 .

Let $M$ and $N$ be two smooth manifolds modeled on the Banach spaces $\mathbb{E}$ and $\mathbb{E}^{\prime}$. Motivated by [28, [7] and [25] we state the following two definitions.

Definition 2.4. Let $g: M \longrightarrow N$ be a smooth map. For $k \in \mathbb{N}$ define the $k$ 'th order differential of $g$ by

$$
\begin{aligned}
T^{k} g: T^{k} M & \longrightarrow T^{k} N \\
{[\gamma, x]_{k} } & \longmapsto[g \circ \gamma, g(x)]_{k}
\end{aligned}
$$

To show that the above definition is well defined consider another representative $[\delta, x]_{k}$ of the class $[\gamma, x]_{k} \in T_{x}^{k} M$. Then, for any integer $1 \leq i \leq k$, using (11) we have

$$
\begin{aligned}
(g \circ \gamma)^{(i)}(0) & =\sum a_{\left(l_{1}, \ldots, l_{j}\right)}^{k} d^{j} g(x)\left(\gamma^{\left(l_{1}\right)}(0), \ldots, \gamma^{\left(l_{j}\right)}(0)\right) \\
& =\sum a_{\left(l_{1}, \ldots, l_{j}\right)}^{k} d^{j} g(x)\left(\delta^{\left(l_{1}\right)}(0), \ldots, \delta^{\left(l_{j}\right)}(0)\right) \\
& =(g \circ \delta)^{(i)}(0)
\end{aligned}
$$

that is $T^{k} g$ is well defined.

Definition 2.5. Let $K_{M}$ and $K_{N}$ be two connection maps on $M$ and $N$ respectively and $g: M \longrightarrow N$ be a smooth map. $K_{M}$ and $K_{N}$ are called $g$-related if they commute with the differentials of $g$ in the following manner

$$
K_{N} \circ T T^{k} g=\oplus_{i=1}^{k} T g \circ K_{M} \text {. }
$$


Remark 2.6. If $k=1$ then, the above definition agrees with that of [28], [7] and [25].

From now on fix the atlas $\mathcal{B}=\left\{\left(V_{\beta}, \psi_{\beta}\right)\right\}_{\beta \in J}$ for $N$ and construct the proposed atlas discussed in theorem 2.1 for $T^{k} N$ which we denote by $\mathcal{B}_{k}=$ $\left\{\left(\pi_{N}^{k-1}\left(V_{\beta}\right), \psi_{\beta}^{k}\right)\right\}_{\beta \in J}$. The model space of $N$ is the Banach space $\mathbb{E}^{\prime}$.

For suitably chosen charts $\left(\pi_{M}^{k}{ }^{-1}\left(U_{\alpha}\right), \phi_{\alpha}^{k}\right)$ and $\left(\pi_{N}^{k-1}\left(V_{\beta}\right), \psi_{\beta}^{k}\right)$, of $M$ and $N$ respectively, we have

$$
\begin{aligned}
& \left(\oplus_{i=1}^{k} \psi_{\beta}^{1}\right) \circ K_{N} \circ T T^{k} g \circ T \phi_{\alpha}^{k^{-1}}= \\
& =\left(\oplus_{i=1}^{k} \psi_{\beta}^{1}\right) \circ K_{N} \circ T \psi_{\beta}^{k^{-1}} \circ T \psi_{\beta}^{k} \circ T T^{k} g \circ T \phi_{\alpha}^{k-1} \\
& :=K_{N, \beta} \circ T T^{k} g_{\beta \alpha}
\end{aligned}
$$

and

$$
\left(\oplus_{i=1}^{k} \psi_{\beta}^{1}\right) \circ\left(\oplus_{i=1}^{k} T g\right) \circ K_{M} \circ T \phi_{\alpha}^{k-1}:=\left(\oplus_{i=1}^{k} T g_{\beta \alpha}\right) \circ K_{M, \alpha}
$$

where $g_{\beta \alpha}:=\psi_{\beta} \circ g \circ \phi_{\alpha}^{-1}$ and $K_{M, \alpha}$ and $K_{N, \beta}$ stand for the local representations of the connection maps pointed out by lemma 2.3 .

In order to reveal the local compatibility condition for $g$-related connections, for any $\left(x, \xi_{1}, \ldots, \xi_{k}, y, \eta_{1}, \ldots, \eta_{k}\right) \in U_{\alpha} \times \mathbb{E}^{2 k+1}$, we define the following auxiliary curve.

$$
\begin{aligned}
\bar{c}:(-\epsilon, \epsilon)^{2} & \longrightarrow \phi_{\alpha}\left(U_{\alpha}\right) \subseteq \mathbb{E} \\
(t, s) & \longmapsto x+s y+\sum_{i=1}^{k} t^{i}\left(\xi_{i}+s \eta_{i}\right)
\end{aligned}
$$

Now, evaluating $\left(\oplus_{i=1}^{k} T g_{\beta \alpha}\right) \circ K_{M, \alpha}$ at $\left(u, y, \eta_{1}, \ldots, \eta_{k}\right)$ yields

$$
\begin{aligned}
& \left(\oplus_{i=1}^{k} T g_{\beta \alpha}\right) \circ K_{M, \alpha}\left(u, y, \eta_{1}, \ldots, \eta_{k}\right)= \\
& \bigoplus_{i=1}^{k}\left(g_{\beta \alpha}(x), d g_{\beta \alpha}(x)\left[\eta_{i}+\stackrel{1}{M} \alpha(u) \eta_{i-1}+\stackrel{2}{M}{ }_{\alpha}(u) \eta_{i-2}+\ldots+\stackrel{i}{M}(u) y\right]\right) .
\end{aligned}
$$

On the other hand

$$
\begin{aligned}
& K_{N, \beta} \circ T T^{k} g_{\beta \alpha}\left(u, y, \eta_{1}, \ldots, \eta_{k}\right) \\
& =\bigoplus_{i=1}^{k}\left(g_{\beta \alpha}(x), \bar{\eta}_{i}+\stackrel{1}{N} \beta(\bar{u}) \bar{\eta}_{i-1}+\cdots+\stackrel{i}{N}{ }_{\beta}(\bar{u}) \bar{y}\right)
\end{aligned}
$$

where $\bar{\gamma}(t):=\bar{c}(t, 0), \bar{u}=\left(\left(g_{\alpha \beta} \circ \bar{\gamma}\right)(0), \ldots, \frac{1}{k !}\left(g_{\alpha \beta} \circ \bar{\gamma}\right)^{(k)}(0)\right), \bar{y}=\frac{\partial}{\partial s}\left(g_{\beta \alpha} \circ\right.$ $\bar{c})(0,0), \bar{\eta}_{i}=\frac{1}{i !} \frac{\partial^{i+1}}{\partial s \partial t^{i}}\left(g_{\beta \alpha} \circ \bar{c}\right)(0,0)$ and $\stackrel{i}{N} \beta$ are the local components of $K_{N}$ for $1 \leq i \leq k$.

With the notation as above, we have the following important compatibility condition which locally declares $g$-related connection maps

$$
\begin{aligned}
& d g_{\beta \alpha}(x)\left[\eta_{i}+\stackrel{1}{M}{ }_{\alpha}(u) \eta_{i-1}+\stackrel{2}{M}{ }_{\alpha}(u) \eta_{i-2}+\ldots+\stackrel{i}{M}(u) y\right] \\
& =\bar{\eta}_{i}+\stackrel{1}{N}_{\beta}(\bar{u}) \bar{\eta}_{i-1}+\cdots+\stackrel{N}{N}_{\beta}(\bar{u}) \bar{y} ; 1 \leq i \leq k .
\end{aligned}
$$

Remark 2.7. If $k=1$, then the last equation coincides with the local compatibility condition for $g$-related connections $K_{M}$ and $K_{N}$ on $M$ and $N$ as it is stated in [28] p. 299. 
Remark 2.8. Whenever $M=N, K_{M}=K_{N}:=K$ and $g=i d_{M}$, the equation (44) reduces to the compatibility condition which locally the connection maps on common charts must satisfy [26].

In what follows, we determine a canonical connection map on $T^{k} M$ depending only on a given linear connection (Riemannian metric) on the base manifold $M$. Keeping the formalisms of [24, 28, 29] we state the following proposition according to [26].

Proposition 2.9. Let $\nabla$ be a linear connection on $M$ with the local components (Christoffel symbols) $\{\Gamma\}_{\alpha \in I}$. There exists an induced connection map on $T^{k} M$ with the following local components.

$$
\begin{aligned}
& \stackrel{1}{M}_{\alpha}\left(x, \xi_{1}\right) y=\Gamma_{\alpha}\left(x, \xi_{1}\right) y \\
& \stackrel{2}{M_{\alpha}}\left(x, \xi_{1}, \xi_{2}\right) y=\frac{1}{2}\left(\sum_{i=1}^{2} \partial_{i} \stackrel{1}{M_{\alpha}}\left(x, \xi_{1}\right)\left(y, i \xi_{i}\right)+\stackrel{1}{M} \alpha\left(x, \xi_{1}\right)\left[\stackrel{1}{M}_{\alpha}\left(x, \xi_{1}\right) y\right]\right) \text {, } \\
& \stackrel{k}{M} \alpha\left(x, \xi_{1}, \ldots, \xi_{k}\right) y=\frac{1}{k}\left(\sum_{i=1}^{k} \partial_{i} \stackrel{k-1}{M}_{\alpha}\left(x, \xi_{1}, \ldots, \xi_{k-1}\right)\left(y, i \xi_{i}\right)\right. \\
& \left.+\stackrel{1}{M}\left(x, \xi_{1}\right)\left[\stackrel{k-1}{M} \alpha\left(x, \xi_{1}, \ldots, \xi_{k-1}\right) y\right]\right) .
\end{aligned}
$$

2.1. Lifting of related linear connections to higher order tangent bundles. In this section we show that lift of $g$-related linear connections remain $g$-related. More precisely let $g: M \longrightarrow N$ be a smooth map between differentiable manifolds $M$ and $N$ and $\nabla_{M}$ and $\nabla_{N}$ be two linear connections on $M$ and $N$ respectively. Moreover suppose that $K_{M}$ and $K_{N}$ be the lifted connection maps (as in proposition [2.9) generated by $\nabla_{M}$ and $\nabla_{N}$ on $T^{k} M$ and $T^{k} N$ respectively.

Proposition 2.10. If $\nabla_{M}$ and $\nabla_{N}$ are g-related, then $K_{M}$ and $K_{N}$ are g-related connection maps too.

Proof. We shall prove that, for any $\alpha \in I, \beta \in J$ with $g\left(U_{\alpha}\right) \subseteq V_{\beta}$, the local components $\left\{\stackrel{i}{M}{ }_{\alpha}\right\}_{i=1, \ldots, k}$ and $\left\{\stackrel{i}{N}_{\beta}\right\}_{i=1, \ldots, k}$ from proposition 2.9 satisfy the condition (44). The proof is by induction on $i$.

For the base step of induction consider the Christoffel symbols $\left\{\Gamma_{\alpha}^{M}\right\}_{\alpha \in I}$ and $\left\{\Gamma_{\beta}^{N}\right\}_{\beta \in J}$ of the $g$ related connections $\nabla_{M}$ and $\nabla_{N}$. The compatibility condition for $\Gamma_{\alpha}^{M}$ and $\Gamma_{\beta}^{N}$ is given by

$$
\begin{aligned}
d g_{\beta \alpha}(x)\left[\eta_{1}+\Gamma_{\alpha}^{M}\left(x, \xi_{1}\right) y\right]= & d g_{\beta \alpha}(x)\left(\eta_{1}\right)+d^{2} g_{\beta \alpha}(x)\left(\xi_{1}, y\right) \\
& +\Gamma_{\beta}^{N}\left(g_{\beta \alpha}(x), d g_{\beta \alpha}(x)\left(\xi_{1}\right)\right) d g_{\beta \alpha}(x)(y)
\end{aligned}
$$

(for more details see [7, 25, 28]). Moreover we have $\stackrel{1}{M}_{\alpha}=\Gamma_{\alpha}^{M}$ and $\stackrel{1}{N_{\beta}}=\Gamma_{\beta}^{N}$. These last three equalities show that $\stackrel{1}{M}_{\alpha}$ and $\stackrel{1}{N}$ 位 satisfy (4). 
By induction, we assume that for $i=1, \ldots k-1, \stackrel{i}{M}$ and $\stackrel{i}{N}_{\beta}$; the rule (44) is verified i.e.

$$
\begin{aligned}
& d g_{\beta \alpha}(x)\left[\eta_{i}+\stackrel{1}{M}{ }_{\alpha}\left(u_{1}\right) \eta_{i-1}+\stackrel{2}{M}{ }_{\alpha}\left(u_{2}\right) \eta_{i-2}+\ldots+\stackrel{i}{M}\left(u_{i}\right) y\right] \\
& =\bar{\eta}_{i}+\stackrel{i}{N}{ }_{\beta}\left(\bar{u}_{1}\right) \bar{\eta}_{i-1}+\cdots+\stackrel{i}{N}_{\beta}\left(\bar{u}_{i}\right) \bar{y}
\end{aligned}
$$

where $u_{j}:=\left(x, \xi_{1}, \ldots, \xi_{j}\right), \bar{u}_{j}=\left(\left(g_{\beta \alpha} \circ \bar{\gamma}\right)(0), \ldots, \frac{1}{j !}\left(g_{\beta \alpha} \circ \bar{\gamma}\right)^{(j)}(0)\right), \bar{y}=$ $\frac{\partial}{\partial s}\left(g_{\beta \alpha} \circ \bar{c}\right)(0,0), \bar{\eta}_{j}=\frac{1}{j !} \frac{\partial^{j+1}}{\partial s \partial t^{j}}\left(g_{\beta \alpha} \circ \bar{c}\right)(0,0)$, for $1 \leq j \leq i$, and $\bar{c}$ and $\bar{\gamma}$ are as in (4). Then from the definition of $M_{\alpha}^{k}$ at $\left(u_{k} ; y, 0, \ldots 0\right)$ we get

$$
\begin{aligned}
k d g_{\beta \alpha}(x) \stackrel{k}{M} \alpha\left(u_{k}\right) y= & d g_{\beta \alpha}(x)\left\{\sum_{i=1}^{k} \partial_{i} \stackrel{k-1}{M} \alpha\left(u_{k-1}\right)\left(y, i \xi_{i}\right)\right. \\
& \left.+\stackrel{1}{M}_{\alpha}\left(x, \xi_{1}\right) \stackrel{k-1}{M}{ }_{\alpha}\left(u_{k-1}\right) y\right\}
\end{aligned}
$$

It perhaps worth remarking that

$$
\begin{aligned}
& d g_{\beta \alpha}(x)\left\{\partial_{1} \stackrel{k-1}{M}{ }_{\alpha}\left(u_{k-1}\right)\left(y, \xi_{1}\right)\right\} \\
& =d g_{\beta \alpha}(x) \frac{d}{d s}(\underbrace{\stackrel{k-1}{M_{\alpha}\left(x+s \xi_{1}, \xi_{1}, \ldots, \xi_{k-1}\right) y}}_{\star}) \\
& =\lim _{s \rightarrow 0}\left(d g_{\beta \alpha}(x) \star-d g_{\beta \alpha}(x) \stackrel{k-1}{M}{ }_{\alpha}\left(u_{k-1}\right) y \pm d g_{\beta \alpha}\left(x+s \xi_{1}\right) \star\right) / s \\
& =-\lim _{s \rightarrow 0}\left(d g_{\beta \alpha}\left(x+s \xi_{1}\right) \star-d g_{\beta \alpha}(x) \star\right) / s \\
& +\lim _{s \rightarrow 0}\left(d g_{\beta \alpha}\left(x+s \xi_{1}\right) \star-d g_{\beta \alpha}(x) \stackrel{k-1}{M}\left(u_{k-1}\right) y\right) / s . \\
& =-d^{2} g_{\beta \alpha}(x)\left(\xi_{1}, \stackrel{k-1}{M} \alpha\left(u_{k-1}\right) y\right) \\
& +\lim _{s \rightarrow 0}\left(d g_{\beta \alpha}\left(x+s \xi_{1}\right) \star-d g_{\beta \alpha}(x) \stackrel{k-1}{M} \alpha\left(u_{k-1}\right) y\right) / s .
\end{aligned}
$$

Now, by the induction hypothesis we have

$$
\begin{aligned}
& d g_{\beta \alpha}(x)\left\{\partial_{1} \stackrel{k-1}{M_{\alpha}}\left(u_{k-1}\right)\left(y, \xi_{1}\right)\right\}=-d^{2} g_{\beta \alpha}(x)\left(\xi_{1}, \stackrel{k-1}{M}_{\alpha}\left(u_{k-1}\right) y\right) \\
& +\frac{\partial}{\partial h}\left\{\frac{\partial^{k}}{(k-1) ! \partial s \partial t^{k-1}}\left(g_{\beta \alpha} \circ \bar{c}_{1}\right)(t, s, h)\right. \\
& +\stackrel{1}{N}_{\beta}\left[\left(g_{\beta \alpha} \circ \bar{\gamma}_{1}\right)(t, h), \frac{\partial}{\partial t}\left(g_{\beta \alpha} \circ \bar{\gamma}_{1}\right)(t, h)\right] \frac{\partial^{k-1}}{(k-2) ! \partial s \partial t^{k-2}}\left(g_{\beta \alpha} \circ \bar{c}_{1}\right)(t, s, h) \\
& +\cdots+\stackrel{k-1}{N}_{\beta}\left[\left(g_{\beta \alpha} \circ \bar{\gamma}_{1}\right)(t, h), \frac{\partial}{\partial t}\left(g_{\beta \alpha} \circ \bar{\gamma}_{1}\right)(t, h), \ldots\right. \\
& \left.\left., \frac{\partial^{k-1}}{(k-1) ! \partial t^{k-1}}\left(g_{\beta \alpha} \circ \bar{\gamma}_{1}\right)(t, h)\right] \frac{\partial}{\partial s}\left(g_{\beta \alpha} \circ \bar{c}_{1}\right)(t, s, h)\right\}\left.\right|_{t=s=h=0}
\end{aligned}
$$


and for $2 \leq i \leq k$

$$
\begin{aligned}
& d g_{\beta \alpha}(x)\left\{\partial_{i} \stackrel{k-1}{M}_{\alpha}\left(u_{k-1}\right)\left(y, i \xi_{i}\right)\right\}=\frac{\partial}{\partial h}\left\{\frac{\partial^{k}}{(k-1) ! \partial s \partial t^{k-1}}\left(g_{\beta \alpha} \circ \bar{c}_{i}\right)(t, s, h)\right. \\
& +\stackrel{1}{N}_{\beta}\left[\left(g_{\beta \alpha} \circ \bar{\gamma}_{i}\right)(t, h), \frac{\partial}{\partial t}\left(g_{\beta \alpha} \circ \bar{\gamma}_{i}\right)(t, h)\right] \frac{\partial^{k-1}}{(k-2) ! \partial s \partial t^{k-2}}\left(g_{\beta \alpha} \circ \bar{c}_{i}\right)(t, s, h) \\
& +\cdots+\stackrel{k-1}{N}_{\beta}\left[\left(g_{\beta \alpha} \circ \bar{\gamma}_{i}\right)(t, h), \frac{\partial}{\partial t}\left(g_{\beta \alpha} \circ \bar{\gamma}_{i}\right)(t, h), \ldots\right. \\
& \left.\left., \frac{\partial^{k-1}}{(k-1) ! \partial t^{k-1}}\left(g_{\beta \alpha} \circ \bar{\gamma}_{i}\right)(t, h)\right] \frac{\partial}{\partial s}\left(g_{\beta \alpha} \circ \bar{c}_{i}\right)(t, s, h)\right\}\left.\right|_{t=s=h=0}
\end{aligned}
$$

where $\bar{c}_{1}(t, s, h):=x+h \xi_{i}+s y+t \xi_{1}+\cdots+t^{k} \xi_{k}, \bar{\gamma}_{1}(t, h)=\bar{c}_{1}(t, 0, h), \ldots$, $\bar{c}_{i}(t, s, h):=x+s y+t \xi_{1}+\cdots+t^{i-2} \xi_{i-2}+t^{i-1}\left(\xi_{i-1}+h i \xi_{i}\right)+t^{i} \xi_{i}+\cdots+t^{k} \xi_{k}$

and $\bar{\gamma}_{i}(t, h)=\bar{c}_{i}(t, 0, h)$. As a result

$$
\begin{aligned}
& k d g_{\beta \alpha}(x) \stackrel{k}{M}\left(u_{k}\right) y \\
= & -d^{2} g_{\beta \alpha}(x)\left(\xi_{1}, \stackrel{k-1}{M}_{\alpha}\left(u_{k-1}\right) y\right)+\sum_{i=1}^{k} \frac{\partial}{\partial h}\left\{\frac{\partial^{k}}{(k-1) ! \partial s \partial t^{k-1}}\left(g_{\beta \alpha} \circ \bar{c}_{i}\right)(t, s, h)\right. \\
& +\stackrel{1}{N}_{\beta}\left[\left(g_{\beta \alpha} \circ \bar{\gamma}_{i}\right)(t, h), \frac{\partial}{\partial t}\left(g_{\beta \alpha} \circ \bar{\gamma}_{i}\right)(t, h)\right] \frac{\partial^{k-1}}{(k-2) ! \partial s \partial t^{k-2}}\left(g_{\beta \alpha} \circ \bar{c}_{i}\right)(t, s, h) \\
& +\cdots+{ }^{k-1} N_{\beta}\left[\left(g_{\beta \alpha} \circ \bar{\gamma}_{i}\right)(t, h), \frac{\partial}{\partial t}\left(g_{\beta \alpha} \circ \bar{\gamma}_{i}\right)(t, h), \ldots\right. \\
& \left.\left., \frac{\partial^{k-1}}{(k-1) ! \partial t^{k-1}}\left(g_{\beta \alpha} \circ \bar{\gamma}_{i}\right)(t, h)\right] \frac{\partial}{\partial s}\left(g_{\beta \alpha} \circ \bar{c}_{i}\right)(t, s, h)\right\}\left.\right|_{t=s=h=0} \\
& +\stackrel{1}{N}_{\beta}\left(\bar{u}_{1}\right)\left[d g_{\beta \alpha}(x) \stackrel{k-1}{M}\left(u_{k-1}\right) y\right]+d^{2} g_{\beta \alpha}(x)\left(\xi_{1}, M_{\alpha}\left(u_{k-1}\right) y\right) .
\end{aligned}
$$

To simplify the above terms, again we use the induction hypothesis for the last line and we get

$$
\begin{aligned}
& k d g_{\beta \alpha}(x) \stackrel{k}{M_{\alpha}}\left(u_{k}\right) y \\
& =\left.\frac{\partial^{k+1}}{(k-1) ! \partial h \partial s \partial t^{k-1}} \sum_{i=1}^{k}\left(g_{\beta \alpha} \circ \bar{c}_{i}\right)(t, s, h)\right|_{t=s=h=0} \\
& +\sum_{j=1}^{2} \partial_{j} \stackrel{1}{N}_{\beta}\left(\bar{u}_{1}\right)\left(\bar{\eta}_{k-2}, \sum_{i=1}^{k}\left\{\frac{\partial^{j}}{(j-1) ! \partial h \partial t^{j-1}}\left(g_{\beta \alpha} \circ \bar{\gamma}_{i}\right)(t, h)\right\}\right)+\ldots \\
& +\left.\sum_{j=1}^{k} \partial_{j} \stackrel{k-1}{N}_{\beta}\left(\bar{u}_{k-1}\right)\left(\bar{y}, \sum_{i=1}^{k}\left\{\frac{\partial^{j}}{(j-1) ! \partial h \partial t^{j-1}}\left(g_{\beta \alpha} \circ \bar{\gamma}_{i}\right)(t, h)\right\}\right)\right|_{t=h=0}
\end{aligned}
$$




$$
\begin{aligned}
& +\stackrel{1}{N}_{\beta}\left(\bar{u}_{1}\right)\left(\left.\frac{\partial^{k}}{(k-2) ! \partial h \partial s \partial t^{k-2}} \sum_{i=1}^{k}\left(g_{\beta \alpha} \circ \bar{c}_{i}\right)(t, s, h)\right|_{t=s=h=0}\right)+\ldots \\
& +\stackrel{k-1}{N}_{\beta}\left(\bar{u}_{k-1}\right)\left(\left.\frac{\partial^{2}}{\partial h \partial s} \sum_{i=1}^{k}\left(g_{\beta \alpha} \circ \bar{c}_{i}\right)(t, s, h)\right|_{t=s=h=0}\right) \\
& +\stackrel{1}{N}_{\beta}\left(\bar{u}_{1}\right)\left[\bar{\eta}_{k-1}+\stackrel{1}{N}_{\beta}\left(\bar{u}_{1}\right) \bar{\eta}_{k-2}+\cdots+\stackrel{k-1}{N}_{\beta}\left(\bar{u}_{k-1}\right) \bar{y}\right]
\end{aligned}
$$

Now, using lemma 6.2, it follows that $k d g_{\beta \alpha}(x) \stackrel{k}{M}{ }_{\alpha}\left(u_{k}\right) y$ is equal to

$$
\begin{aligned}
& k \bar{\eta}_{k}+\sum_{j=1}^{2} \partial_{j} \stackrel{1}{N}_{\beta}\left(\bar{u}_{1}\right)\left(\bar{\eta}_{k-2}, j \bar{\xi}_{j}\right)+\cdots+\sum_{j=1}^{k} \partial_{j} \stackrel{k-1}{N}_{\beta}\left(\bar{u}_{k-1}\right)\left(\bar{y}, j \bar{\xi}_{j}\right) \\
& +(k-1) \stackrel{1}{N}_{\beta}\left(\bar{u}_{1}\right) \bar{\eta}_{k-1}+\cdots+\stackrel{k-1}{N}_{\beta}\left(\bar{u}_{k-1}\right) \bar{\eta}_{1} \\
& +\stackrel{1}{N} \beta\left(\bar{u}_{1}\right)\left[\bar{\eta}_{k-1}+\stackrel{1}{N}{ }_{\beta}\left(\bar{u}_{1}\right) \bar{\eta}_{k-2}+\cdots+\stackrel{k-1}{N} \beta\left(\bar{u}_{k-1}\right) \bar{y}\right] \\
& =k \bar{\eta}_{k}+2 \stackrel{2}{N}{ }_{\beta}\left(\bar{u}_{2}\right) \bar{\eta}_{k-2}+\cdots+k \stackrel{k}{N}{ }_{\beta}\left(\bar{u}_{k}\right) \bar{y}+(k-1) \stackrel{1}{N}{ }_{\beta}\left(\bar{u}_{1}\right) \bar{\eta}_{k-1} \\
& +(k-2) \stackrel{2}{N_{\beta}}\left(\bar{u}_{2}\right) \bar{\eta}_{k-2}+\cdots+\stackrel{k-1}{N}_{\beta}\left(\bar{u}_{k-1}\right) \bar{\eta}_{1}+\stackrel{1}{N}_{\beta}\left(\bar{u}_{1}\right)\left(\bar{\eta}_{k-1}\right) \\
& =k \bar{\eta}_{k}+(1+(k-1)) \stackrel{1}{N}{ }_{\beta}\left(\bar{u}_{1}\right) \bar{\eta}_{k-1}+\cdots+((k-1)+1) \stackrel{k-1}{N}{ }_{\beta}\left(\bar{u}_{k-1}\right) \bar{\eta}_{1} \\
& +k \stackrel{k}{N}{ }_{\beta}\left(\bar{u}_{k}\right) \bar{y} \\
& =k\left(\bar{\eta}_{k}+\stackrel{1}{N}{ }_{\beta}\left(\bar{u}_{1}\right) \bar{\eta}_{k-1}+\cdots+\stackrel{k}{N}{ }_{\beta}\left(\bar{u}_{k}\right) \bar{y}\right)
\end{aligned}
$$

where $\bar{\xi}_{j}=\operatorname{Proj}_{j+1}\left(\bar{u}_{k}\right)$ and $\operatorname{Proj}_{j+1}, 0 \leq j \leq k-1$, is the projection map to the $(j+1)$ 'th factor. As a consequence we obtain

$$
d g_{\beta \alpha}(x) \stackrel{k}{M}{ }_{\alpha}\left(x, \xi_{1}, \ldots, \xi_{k}\right) y=\bar{\eta}_{k}+\stackrel{1}{N}{ }_{\beta}\left(\bar{u}_{1}\right) \bar{\eta}_{k-1}+\cdots+\stackrel{k}{N}{ }_{\beta}\left(\bar{u}_{k}\right) \bar{y} .
$$

However, as we are about to see, the desired compatibility condition is finally handled. More precisely we have shown that for the vector bundle morphisms $K_{M}$ and $K_{N}$ locally

$$
\oplus_{i=1}^{k} T g_{\beta \alpha}(x) K_{M, \alpha}(u ; y, 0, \ldots, 0)=K_{N, \beta} \circ T T^{k} g_{\beta \alpha}(u ; y, 0, \ldots, 0) .
$$

Moreover by the induction hypothesis we have $\oplus_{i=1}^{k} T g_{\beta \alpha}(x) K_{M, \alpha}\left(u ; 0, \eta_{1}, \eta_{2}, \ldots, \eta_{k}\right)=K_{N, \beta} \circ T T^{k} g_{\beta \alpha}\left(u ; 0, \eta_{1}, \eta_{2}, \ldots, \eta_{k}\right)$.

Since $K_{M}$ and $K_{N}$ are vector bundle morphisms (and linear on fibres) we can add the last two equalities. As a consequence we have

$\oplus_{i=1}^{k} T g_{\beta \alpha}(x) K_{M, \alpha}\left(u ; y, \eta_{1}, \eta_{2}, \ldots, \eta_{k}\right)=K_{N, \beta} \circ T T^{k} g_{\beta \alpha}\left(u ; y, \eta_{1}, \eta_{2}, \ldots, \eta_{k}\right)$.

This last means that $K_{M}$ and $K_{N}$ are $g$-related connection maps on the $k$ 'th order tangent bundles of $M$ and $N$ respectively, constructed only with the help of the connections $\nabla_{M}$ and $\nabla_{N}$. 
2.2. $T^{k} M$ as a vector bundle. For $k \geq 2$, the bundle structure defined in theorem 2.1 is quite far from being a vector bundle due to the complicated nonlinear transition functions. However, according to [26] we have the following main theorem.

Theorem 2.11. Let $\nabla$ be a linear connection on $M$ and $K$ the induced connection map introduced in proposition 2.9. The following trivializations define a vector bundle structure on $\pi_{M}^{k}: T^{k} M \longrightarrow M$ with the structure group $G L\left(\mathbb{E}^{k}\right)$.

$$
\begin{aligned}
\Phi_{\alpha}^{k}: \pi_{M}^{k-1}\left(U_{\alpha}\right) & \longrightarrow \phi_{\alpha}\left(U_{\alpha}\right) \times \mathbb{E}^{k} \\
{[\gamma, x]_{k} } & \longmapsto\left(\gamma_{\alpha}(0), \gamma_{\alpha}^{(1)}(0), z^{2}\left([\gamma, x]_{k}\right), \ldots, z^{k}\left([\gamma, x]_{k}\right)\right)
\end{aligned}
$$

where $\gamma_{\alpha}=\phi_{\alpha} \circ \gamma$ and

$$
\begin{aligned}
z_{\alpha}^{2}\left([\gamma, x]_{k}\right)= & \frac{1}{2}\left\{\frac{\gamma_{\alpha}^{(2)}(0)}{1 !}+M_{\alpha}\left[\gamma_{\alpha}(0), \gamma_{\alpha}^{(1)}(0)\right] \gamma_{\alpha}^{(1)}(0)\right\} \\
\vdots & \\
z_{\alpha}^{k}\left([\gamma, x]_{k}\right)= & \frac{1}{k}\left\{\frac{\gamma_{\alpha}^{(k)}(0)}{(k-1) !}+M_{\alpha}\left[\gamma_{\alpha}(0), \gamma_{\alpha}^{(1)}(0)\right] \frac{\gamma_{\alpha}^{(k-1)}(0)}{(k-2) !}+\ldots\right. \\
& \left.+\stackrel{k-1}{M}{ }_{\alpha}\left[\gamma_{\alpha}(0), \gamma_{\alpha}^{(1)}(0), \ldots, \frac{\gamma_{\alpha}^{(k-1)}(0)}{(k-1) !}\right] \gamma_{\alpha}^{(1)}(0)\right\} .
\end{aligned}
$$

Moreover setting $\Phi_{\beta \alpha}^{k}=\Phi_{\beta}^{k} \circ \Phi_{\alpha}^{k-1}, \phi_{\beta \alpha}=\phi_{\beta} \circ \phi_{\alpha}^{-1}$ and $U_{\beta \alpha}=U_{\alpha} \cap U_{\beta}$ the transition map

$$
\Phi_{\beta \alpha}^{k}: U_{\beta \alpha} \longrightarrow G L\left(\mathbb{E}^{k}\right)
$$

is given by $\Phi_{\beta \alpha}^{k}(x)\left(\xi_{1}, \xi_{2}, \ldots, \xi_{k}\right)=\left(\phi_{\beta \alpha}(x), d \phi_{\beta \alpha}(x) \xi_{1}, \ldots, d \phi_{\beta \alpha}(x) \xi_{k}\right)$ that is $T^{k} M$, as a vector bundle, is isomorphic to $\oplus_{i=1}^{k} T M$.

The converse of the above theorem is also true i.e. if $\pi_{M}^{k}: T^{k} M \longrightarrow M$, for some $k \geq 2$, admits a vector bundle structure isomorphic to $\oplus_{i=1}^{k} T M$, then a linear connection on $M$ can be defined [26].

Remark 2.12. One can replace the induced connection map in theorem 2.11 with a general connection map (in the sense of definition 2.2) and prove the theorem in a similar fashion. One reason for using this rather elaborate model (induced connection maps) is that it permits a concrete way of constructing.

Remark 2.13. For $i<k$

$$
\begin{aligned}
& \pi_{M}^{k, i}: T^{k} M \quad \longrightarrow T^{i} M \\
& {[\gamma, x]_{k} \longmapsto[\gamma, x]_{i}}
\end{aligned}
$$

also admits a vector bundle structure [26].

Remark 2.14. If the base manifold $M$ is $C^{k}$-partitionable, then the existence of a connection on $M$ and equivalently a vector bundle structure on $\pi_{M}^{k}: T^{k} M \longrightarrow M$ is guaranteed (see also [25] p. 94).

However, existence of a $C^{k}$ partition of unity on the discussing manifolds puts some restrictions on the model spaces. According to [15] if $M$ is a 
paracompact $C^{k}$ manifold modeled on a separable $S C^{p}$ Banach space with $p \geq \max \{2, k\}$, then $M$ admits a $C^{k}$ partition of unity. As a corollary every paracompact $C^{k}$ manifold modeled on a separable Hilbert space admits a $C^{k}$ partition of unity [14, 15, 16].

As we have shown, the vector bundle structure on $T^{k} M$ depends heavily on the chosen linear connection (see e.g. example 5.2). In the next section we ask about the extent to which this vector bundle structure remains isomorphic.

\section{3. $T^{k} g$ AS A VECTOR BUNDLE MORPHISM}

For a differentiable map $g: M \longrightarrow N$, in contrast to $T^{1} g=T g: T M \longrightarrow$ $T N$, the tangent map $T^{k} g$, even for $k=2$, is not necessarily a vector bundle morphism [7, 25]. In this section first, we investigate under what conditions $T^{k} g$ becomes a vector bundle morphism.

Let $K_{M}$ and $K_{N}$ be two connection maps on $T^{k} M$ and $T^{k} N$ respectively, possibly, induced by linear connection $\nabla_{M}$ and $\nabla_{N}$. For $[\gamma, x]_{k} \in T^{k} M$ suppose that $\left(\pi_{M}^{k-1}\left(U_{\alpha}\right), \Phi_{\alpha}^{k}\right)$ and $\left(\pi_{N}^{k-1}\left(V_{\beta}\right), \Psi_{\beta}^{k}\right)$ be two vector bundle trivializations (given by theorem 2.11) around $x \in M$ and $g(x) \in N$ respectively. For $\left(x, \xi_{1}, \ldots, \xi_{k}\right) \in U_{\alpha} \times \mathbb{E}^{k}$, define the curve $\bar{\mu}_{k}$ inductively as follows; $\bar{\mu}_{1}(t)=x+t \xi_{1}, \bar{\mu}_{2}(t)=\bar{\mu}_{1}(t)+\frac{t^{2}}{2}\left\{2 \xi_{2}-\stackrel{1}{M}_{\alpha}\left(x, \xi_{1}\right) \xi_{1}\right\}$ and for $i \geq 2$,

$$
\begin{gathered}
\bar{\mu}_{i}(t)=\bar{\mu}_{i-1}(t)+\frac{t^{i}}{i}\left\{i \xi_{i}-M_{\alpha}\left(x, \xi_{1}\right) \frac{\bar{\mu}_{i-1}^{(i-1)}(0)}{(i-2) !}-\ldots\right. \\
\left.\stackrel{i-1}{\alpha}_{\alpha}\left(x, \xi_{1}, \frac{1}{2 !} \bar{\mu}_{i-1}^{(2)}(0), \ldots, \frac{1}{(i-1) !} \bar{\mu}_{i-1}^{(i-1)}(0)\right) \xi_{1}\right\} .
\end{gathered}
$$

In order to reduce the intricate computations as much as possible, we will use the following lemma.

Lemma 3.1. Fix the positive integer $k \geq 2$ and suppose that $\bar{\mu}:=\bar{\mu}_{k}$ be the curve defined above, $\mathcal{O} \subseteq \mathbb{E}$ be open and $f: \mathcal{O} \longrightarrow \mathbb{E}$ be any smooth map. Then

$$
\left.\frac{\partial^{k}}{\partial s \partial t^{k-1}}\left(f \circ \bar{d}_{k}\right)(t, s)\right|_{t=s=0}=\left.(f \circ \bar{\mu})^{(k)}(t)\right|_{t=0}
$$

where

$$
\bar{d}_{k}:(-\epsilon, \epsilon)^{2} \longrightarrow \mathbb{E} ;(t, s) \longmapsto \sum_{i=1}^{k-1} \frac{t^{i}}{i !}\left(\bar{\mu}^{(i)}(0)+s \bar{\mu}^{(i+1)}(0)\right) .
$$

Proof. See appendix.

Now, suppose that $\mu:=\phi_{\alpha}^{-1} \circ \bar{\mu}$. Then the local representation of $T^{k} g$ is $\Psi_{\beta}^{k} \circ T^{k} g \circ \Phi_{\alpha}^{k-1}\left(x, \xi_{1}, \ldots, \xi_{k}\right)=\Psi_{\beta}^{k} \circ T^{k} g\left([\mu, x]_{k}\right)=\Psi_{\beta}^{k}\left([g \circ \mu, g(x)]_{k}\right)$.

With the fact $\psi_{\beta} \circ g \circ \mu=\psi_{\beta} \circ g \circ \phi_{\alpha}^{-1} \circ \phi_{\alpha} \circ \mu:=g_{\beta \alpha} \circ \bar{\mu}$ in mind, we apply the vector bundle trivialization of theorem 2.11 to $[g \circ \mu, g(x)]_{k}$ and we get

$$
\Psi_{\beta}^{k}\left([g \circ \mu, g(x)]_{k}\right)=\left(g_{\beta \alpha}(x), d g_{\beta \alpha}(x) \xi_{1}, z_{\beta}^{1}\left([\mu \circ g, g(x)]_{k}\right), \ldots, z_{\beta}^{k}\left([\mu \circ g, g(x)]_{k}\right)\right)
$$


However, for $2 \leq i \leq k$ we have

$$
\begin{aligned}
& i z_{\beta}^{i}\left([g \circ \mu, g(x)]_{k}\right) \\
& =d g_{\beta \alpha}(x)\left[i \xi_{i}-M_{\alpha}^{1}\left(v_{1}\right) \frac{(\bar{\mu})^{(i-1)}(0)}{(i-2) !}-\cdots-\stackrel{i-1}{M}_{\alpha}\left(v_{i-1}\right)(\bar{\mu})^{(1)}(0)\right] \\
& +\frac{1}{(i-1) !}\left\{\sum_{l_{1}+l_{2}=i} a_{\left(l_{1}, l_{2}\right)}^{i} d^{2} g_{\beta \alpha}(x)\left((\bar{\mu})^{\left(l_{1}\right)}(0),(\bar{\mu})^{\left(l_{2}\right)}(0)\right)+\ldots\right. \\
& \left.+d^{i} g_{\beta \alpha}(x)\left(\left(\bar{\mu}^{(1)}\right)(0), \ldots,\left(\bar{\mu}^{(1)}\right)(0)\right)\right\} \\
& +\stackrel{1}{N}_{\beta}\left(\bar{v}_{1}\right) \frac{\left(g_{\beta \alpha} \circ \bar{\mu}\right)^{(i-1)}(0)}{(i-2) !}+\cdots+\stackrel{i-1}{N}_{\beta}\left(\bar{v}_{i-1}\right)\left(g_{\beta \alpha} \circ \bar{\mu}\right)^{(1)}(0)
\end{aligned}
$$

where $v=\left(\bar{\mu}(0), \bar{\mu}^{(1)}(0), \ldots, \frac{\bar{\mu}^{k}(0)}{k !}\right), v_{j}=\operatorname{Proj}_{j+1}(v)$,

$$
\begin{aligned}
\bar{v} & =\left(\left(g_{\beta \alpha} \circ \bar{d}\right)(0,0), \frac{\partial}{\partial t}\left(g_{\beta \alpha} \circ \bar{d}\right)(0,0), \ldots, \frac{\partial^{k}}{\partial t^{k}} \frac{\left(g_{\beta \alpha} \circ \bar{d}\right)(0,0)}{k !}\right) \\
& =\left(\left(g_{\beta \alpha} \circ \bar{\mu}\right)(0),\left(g_{\beta \alpha} \circ \bar{\mu}\right)^{(1)}(0), \ldots, \frac{\left(g_{\beta \alpha} \circ \bar{\mu}\right)^{(k)}(0)}{k !}\right)
\end{aligned}
$$

and $\bar{v}_{j}=\operatorname{Proj}_{j+1}(\bar{v})$.

As we can see now, due to the presence of higher order derivatives, as $k$ increases, (generally) it becomes increasingly difficult for $T^{k} g$ to be a vector bundle morphism.

To get around this difficulty, let $K_{M}$ and $K_{N}$ be connection maps which are induced by linear connections $\nabla_{M}$ and $\nabla_{N}$ respectively.

Theorem 3.2. If $\nabla_{M}$ and $\nabla_{N}$ are g-related then, $T^{k} g: T^{k} M \longrightarrow T^{k} N$ becomes a vector bundle morphism.

Proof. If $[\mu, x]_{k} \in T_{x}^{k} M$, then $\pi_{N}^{k} \circ T^{k} g\left([\mu, x]_{k}\right)=\pi_{N}^{k}\left([g \circ \mu, g(x)]_{k}\right)=g(x)=$ $g \circ \pi_{M}^{k}\left([\mu, x]_{k}\right)$ means that $T^{k} g$ is fibre preserving. Now consider the trivializations $\left(\pi_{M}^{k-1}\left(U_{\alpha}\right), \Phi_{\alpha}^{k}\right)$ and $\left(\pi_{N}^{k-1}\left(V_{\beta}\right), \Psi_{\beta}^{k}\right)$, as introduced in theorem 2.11, around $x$ and $g(x)$ respectively. Our main task is to show that $\Psi_{\beta}^{k} \circ T^{k} g \circ \Phi_{\alpha}^{k^{-1}}$ is linear on fibres.

Step 1. Setting $\eta_{i}:=\frac{1}{(i-1) !} \bar{\mu}^{(i)}(0), y:=\bar{\mu}^{(1)}(0)$ and $\xi_{i}:=\frac{1}{i !} \bar{\mu}^{(i)}(0)$ in compatibility condition (4), and using lemma 3.1 with $g_{\beta \alpha}=f$ we get

$$
\begin{gathered}
d g_{\beta \alpha}(x) \frac{\bar{\mu}^{(i)}(0)}{(i-1) !}+d g_{\beta \alpha}(x) \stackrel{1}{M_{\alpha}}\left(v_{1}\right) \frac{\bar{\mu}^{(i-1)}(0)}{(i-2) !} \cdots+d g_{\beta \alpha}(x) \stackrel{i-1}{M_{\alpha}}\left(v_{i-1}\right) \bar{\mu}^{(1)}(0) \\
=\frac{\partial^{i}}{\partial s \partial t^{i-1}} \frac{\left(g_{\beta \alpha} \circ \bar{d}_{k}\right)(0,0)}{(i-1) !}+\stackrel{1}{N}_{\beta}\left(\bar{v}_{1}\right) \frac{\partial^{i-1}}{\partial s \partial t^{i-2}} \frac{\left(g_{\beta \alpha} \circ \bar{d}_{k}\right)(0,0)}{(i-2) !}+ \\
\cdots+\stackrel{i-1}{N}_{\beta}\left(\bar{v}_{i-1}\right) \frac{\partial}{\partial s}\left(g_{\beta \alpha} \circ \bar{d}_{k}\right)(0,0) \\
=\frac{\left(g_{\beta \alpha} \circ \bar{\mu}\right)^{(i)}(0)}{(i-1) !}+\stackrel{1}{N}_{\beta}\left(\bar{v}_{1}\right) \frac{\left(g_{\beta \alpha} \circ \bar{\mu}\right)^{(i-1)}(0)}{(i-2) !}+\cdots+\stackrel{i-1}{N}_{\beta}\left(\bar{v}_{i-1}\right)\left(g_{\beta \alpha} \circ \bar{\mu}\right)^{(1)}(0) .
\end{gathered}
$$


As a consequence we obtain

$$
\begin{aligned}
& \stackrel{1}{N}_{\beta}\left(\bar{v}_{1}\right) \frac{\left(g_{\beta \alpha} \circ \bar{\mu}\right)^{(i-1)}(0)}{(i-2) !}+\ldots \stackrel{i-1}{N} \beta\left(\bar{v}_{i-1}\right)\left(g_{\beta \alpha} \circ \bar{\mu}\right)^{(1)}(0) \\
& =d g_{\beta \alpha}(x) \frac{\bar{\mu}^{(i)}(0)}{(i-1) !}+d g_{\beta \alpha}(x) \stackrel{1}{M} \alpha\left(v_{1}\right) \frac{\bar{\mu}^{(i-1)}(0)}{(i-2) !}+\ldots \\
& \quad+d g_{\beta \alpha}(x) \stackrel{i-1}{M}{ }_{\alpha}\left(v_{i-1}\right) \bar{\mu}^{(1)}(0)-\frac{\left(g_{\beta \alpha} \circ \bar{\mu}\right)^{(i)}(0)}{(i-1) !} .
\end{aligned}
$$

Step 2. We now apply the previous observation to (8) and we get

$$
\begin{gathered}
i z_{\beta}^{k}\left([g \circ \mu, g(x)]_{k}\right) \\
=i d g_{\beta \alpha}(x) \xi_{i}+\frac{1}{(i-1) !}\left\{\sum_{l_{1}+l_{2}=i} a_{\left(l_{1}, l_{2}\right)}^{i} d^{2} g_{\beta \alpha}(x)\left((\bar{\mu})^{\left(l_{1}\right)}(0),(\bar{\mu})^{\left(l_{2}\right)}(0)\right)\right. \\
\left.+\cdots+d^{i} g_{\beta \alpha}(x)\left(\left(\bar{\mu}^{(1)}\right)(0), \ldots,\left(\bar{\mu}^{(1)}\right)(0)\right)\right\}+d g_{\beta \alpha}(x) \frac{\bar{\mu}^{(i)}(0)}{(i-1) !} \\
-\frac{\left(g_{\beta \alpha} \circ \bar{\mu}\right)^{(i)}(0)}{(i-1) !} \\
=i d g_{\beta \alpha}(x) \xi_{i}+\frac{1}{(i-1) !}\left\{\sum_{l_{1}+l_{2}=i} a_{\left(l_{1}, l_{2}\right)}^{i} d^{2} g_{\beta \alpha}(x)\left((\bar{\mu})^{\left(l_{1}\right)}(0),(\bar{\mu})^{\left(l_{2}\right)}(0)\right)\right. \\
\left.+\cdots+d^{i} g_{\beta \alpha}(x)\left(\left(\bar{\mu}^{(1)}\right)(0), \ldots,\left(\bar{\mu}^{(1)}\right)(0)\right)\right\}+d g_{\beta \alpha}(x) \frac{\bar{\mu}^{(i)}(0)}{(i-1) !} \\
-\frac{1}{(i-1) !}\left\{d g_{\beta \alpha}(x) \bar{\mu}^{(i)}(0)+\sum_{l_{1}+l_{2}=i} a_{\left(l_{1}, l_{2}\right)} d^{2} g_{\beta \alpha}(x)\left((\bar{\mu})^{\left(l_{1}\right)}(0)\right.\right. \\
\left.\left.,(\bar{\mu})^{\left(l_{2}\right)}(0)\right)+\cdots+d^{i} g_{\beta \alpha}(x)\left(\left(\bar{\mu}^{(1)}\right)(0), \ldots,\left(\bar{\mu}^{(1)}\right)(0)\right)\right\} \\
=i d g_{\beta \alpha}(x) \xi_{i} .
\end{gathered}
$$

As a consequence we have

$\Psi_{\beta}^{k} \circ T^{k} g \circ \Phi_{\alpha}^{k-1}\left(x, \xi_{1}, \ldots, \xi_{k}\right)=\left(g_{\beta \alpha}(x), d g_{\beta \alpha}(x)\left(\xi_{1}\right), \ldots, d g_{\beta \alpha}(x)\left(\xi_{k}\right)\right)$.

This last means that $T^{k} g$ is fibre linear and

$$
\begin{aligned}
T^{k} g_{\beta \alpha}: U_{\alpha} & \longrightarrow \mathcal{L}\left(\mathbb{E}^{k}, \mathbb{E}^{\prime k}\right) \\
x & \longmapsto \Psi_{\beta, g(x)}^{k} \circ T_{x}^{k} g \circ \Phi_{\alpha, x}^{k-1}
\end{aligned}
$$

is a smooth morphism which completes the proof.

Remark 3.3. One can replace the induced $g$-related connection maps with general $g$-related connection maps and prove theorem 3.2 in an exactly similar way.

Remark 3.4. If $M=N$ and $g=i d_{M}$ then theorem 3.2 yields

$$
\begin{aligned}
\Phi_{\beta}^{k} \circ \Phi_{\alpha}^{k^{-1}}: U_{\alpha} & \longrightarrow \mathcal{L}\left(\mathbb{E}^{k}, \mathbb{E}^{k}\right) \\
x & \longmapsto\left(d \phi_{\beta} \circ \phi_{\alpha}^{-1}(x)(.), \ldots, d \phi_{\beta} \circ \phi_{\alpha}^{-1}(x)(.)\right)
\end{aligned}
$$

as it was noted by theorem 3.3 of [26].

The next corollary is a direct consequence of theorems 3.2 and 2.10 .

Corollary 3.5. If $g$ is a diffeomorphism, then $T^{k} g$ becomes a vector bundle isomorphism. 
Remark 3.6. Let $\nabla_{M}$ and $\nabla_{N}$ be two $g$-related connections on $M$ and $N$ respectively. Consider the vector bundle structures on $\left(T^{k} M, \pi_{M}^{k, i}, T^{i} M\right)$ and $\left(T^{k} N, \pi_{N}^{k, i}, T^{i} N\right)$ proposed by theorem 2.11. Then the argument of theorem 3.2 can easily be modified to prove

$$
\left(T^{k} g, T^{i} g\right):\left(T^{k} M, \pi_{M}^{k, i}, T^{i} M\right) \longrightarrow\left(T^{k} N, \pi_{N}^{k, i}, T^{i} N\right)
$$

is also a vector bundle morphism for $i<k$.

\section{4. $T^{\infty} g$ AS A VECTOR BUNDLE MORPHISM}

As we have seen in the previous sections, at the presence of $g$-related connections on $M$ and $N$ for any $k \in \mathbb{N},\left(T^{k} g, g\right)$ becomes a vector bundle morphism. If we take one step further by considering $T^{\infty} M$ and $T^{\infty} N$, as generalized Fréchet vector bundles over $M$ and $N$ respectively (26]), then proving $T^{\infty} g$ to be a generalized vector bundle morphism becomes much more complicated.

More precisely the set of linear maps between $\mathbb{F}:=\varliminf_{\longleftarrow} \mathbb{E}^{k}$ and $\mathbb{F}^{\prime}:=$ $\lim _{\mathbb{E}^{\prime}}$ (the fibre types of $\left(T^{\infty} M, \pi_{M}^{\infty}, M\right)$ and $\left(T^{\infty} N, \pi_{N}^{\infty}, N\right)$ respectively) does not remain in the category of Fréchet spaces [12, 22 .

In this section employing the projective limit methodology, as in 1, 11, 26, 27] etc., we show that at the presence of $g$-related connections on $M$ and $N,\left(T^{\infty} g, g\right)$ becomes a vector bundle morphism.

Of course one can consider a projective system of $g$-related connection maps on $T^{k} M$ and $T^{k} N, k \in \mathbb{N}$ and prove the same results.

Let the notation be as in the preceding sections and for the natural numbers $j \geq i$ consider the projections $\pi_{M}^{j, i}: T^{j} M \longrightarrow T^{i} M$ and $\pi_{N}^{j, i}$ : $T^{j} N \longrightarrow T^{i} N$ mapping $[\gamma, x]_{j}$ onto $[\gamma, x]_{i}$ as connecting morphisms of the projective families $\left\{T^{k} M\right\}_{k \in \mathbb{N}}$ and $\left\{T^{k} N\right\}_{k \in \mathbb{N}}$ (for more details see [26], [27]. The family $\left\{T^{k} g\right\}_{k \in \mathbb{N}}$ form a projective system of maps since $\pi_{N}^{j, i} \circ T^{j} g=$ $T^{i} g \circ \pi_{M}^{j, i}$. More precisely

$$
\pi_{N}^{j, i} \circ T^{j} g\left([\gamma, x]_{j}\right)=\pi_{N}^{j, i}\left([g \circ \gamma, g(x)]_{j}\right)=[g \circ \gamma, g(x)]_{i}
$$

and

$$
T^{i} g \circ \pi_{M}^{j, i}\left([\gamma, x]_{j}\right)=T^{i} g\left([\gamma, x]_{i}\right)=[g \circ \gamma, g(x)]_{i} .
$$

As a consequence the limit map $T^{\infty} g:=\lim T^{k} g$ exists and maps the thread $\left([\gamma, x]_{k}\right)_{k \in \mathbb{N}} \in T^{\infty} M:=\lim _{\longleftarrow} T^{k} M$ to $\left([g \circ \gamma, x]_{k}\right)_{k \in \mathbb{N}} \in T^{\infty} N=\lim _{\longleftarrow} T^{k} N$. It is easily checked that, for any $x \in M$, the families $\left\{\Phi_{\alpha, x}^{k}\right\}_{k \in \mathbb{N}}$ and $\left\{\Psi_{\beta, g(x)}^{k}\right\}_{k \in \mathbb{N}}$, as in theorem 2.11, form projective systems of trivializations with the limits $\Phi_{\alpha, x}^{\infty}$ and $\Psi_{\beta, g(x)}^{\infty}$ respectively (see also [1]).

But $T^{\infty} g$ seems to be far from being called a vector bundle morphism due to the difficulties emerged in $\mathcal{L}\left(\mathbb{F}, \mathbb{F}^{\prime}\right)$ and therefore the problematic map

$$
\begin{aligned}
T^{\infty} g_{\beta \alpha}: U_{\alpha} & \longrightarrow \mathcal{L}\left(\mathbb{F}, \mathbb{F}^{\prime}\right) \\
x & \longmapsto \Psi_{\beta, g(x)}^{\infty} \circ T^{\infty} \circ g \circ \Phi_{\alpha, x}^{\infty} .
\end{aligned}
$$


To overcome this obstacle define

$$
\mathcal{H}\left(\mathbb{F}, \mathbb{F}^{\prime}\right)=\left\{\left(l_{k}\right)_{k \in \mathbb{N}} \in \prod_{k=1}^{\infty} \mathcal{L}\left(\mathbb{E}^{k}, \mathbb{E}^{\prime k}\right): \rho_{j i}^{\prime} \circ l_{j}=l_{i} \circ \rho_{j i}, \forall j \geq i\right\}
$$

where $\rho_{j i}: \mathbb{E}^{j} \longrightarrow \mathbb{E}^{i}$ and $\rho_{j i}^{\prime}: \mathbb{E}^{\prime j} \longrightarrow \mathbb{E}^{\prime i}$ are the projection maps to the first $i$ factors. $\mathcal{H}\left(\mathbb{F}, \mathbb{F}^{\prime}\right)$ is a Fréchet space $([1])$ isomorphic to the projective limit of the projective system of Banach spaces $\left\{\mathcal{H}^{k}\left(\mathbb{E}^{k}, \mathbb{E}^{\prime k}\right)\right\}_{k \in \mathbb{N}}$ where

$\mathcal{H}^{k}\left(\mathbb{E}^{k}, \mathbb{E}^{\prime k}\right)=\left\{\left(l_{i}\right)_{1 \leq i \leq k} \in \prod_{i=1}^{k} \mathcal{L}\left(\mathbb{E}^{i}, \mathbb{E}^{\prime i}\right): \rho_{j i}^{\prime} \circ l_{j}=l_{i} \circ \rho_{j i}, \quad \forall 1 \leq i \leq j \leq k\right\}$.

However, for any $\xi_{1}, \ldots, \xi_{j} \in \mathbb{E}$ and $j \geq i$ we have

$$
\begin{aligned}
& \rho_{j i}^{\prime} \circ\left(\Psi_{\beta, g(x)}^{j} \circ T^{j} g \circ \Phi_{\alpha, x}^{j}{ }^{-1}\right)\left(\xi_{1}, \ldots, \xi_{j}\right)=\rho_{j i}^{\prime}\left(d g_{\beta \alpha}(x) \xi_{1}, \ldots, d g_{\beta \alpha}(x) \xi_{j}\right) \\
& =\left(d g_{\beta \alpha}(x) \xi_{1}, \ldots, d g_{\beta \alpha}(x) \xi_{i}\right)=\left(\Psi_{\beta, g(x)}^{i} \circ T^{i} g \circ \Phi_{\alpha, x}^{i}{ }^{-1}\right) \circ \rho_{j i}\left(\xi_{1}, \ldots, \xi_{j}\right)
\end{aligned}
$$

meaning that $\left\{\Psi_{\beta, g(x)}^{k} \circ T^{k} g \circ \Phi_{\alpha, x}^{k}{ }^{-1}\right\}_{k \in \mathbb{N}}$, with the limit $\Psi_{\beta, g(x)}^{\infty} \circ T^{\infty} g \circ$ $\Phi_{\alpha, x}^{\infty}{ }^{-1}$, belongs to $\mathcal{H}\left(\mathbb{F}, \mathbb{F}^{\prime}\right)$. As a consequence $T^{\infty} g_{\beta \alpha}: U_{\alpha} \longrightarrow \mathcal{L}\left(\mathbb{F}, \mathbb{F}^{\prime}\right)$; $x \longmapsto \varepsilon \circ \Psi_{\beta, g(x)}^{\infty} \circ T^{\infty} g \circ \Phi_{\alpha, x}^{\infty}{ }^{-1}$ is smooth (in the sense of Leslie and Galanis [18, 19, 10, 11]) where $\varepsilon$ is the linear (and smooth) map defined by

$$
\begin{aligned}
\varepsilon: \mathcal{H}\left(\mathbb{F}, \mathbb{F}^{\prime}\right) & \longrightarrow \mathcal{L}\left(\mathbb{F}, \mathbb{F}^{\prime}\right) \\
\left(l_{k}\right)_{k \in \mathbb{N}} & \longmapsto \lim _{\leftarrow} l_{k} .
\end{aligned}
$$

As a result, $T^{\infty} g$ is a generalized smooth map.

Remark 4.1. It is known that differential calculus in normed spaces does not have a unique canonical extension to general topological spaces (For a list of definitions see e.g. [3, 13]).

In our case, the problem is due to $\mathcal{L}\left(\mathbb{F}, \mathbb{F}^{\prime}\right)$ which is not normable. In fact $\mathcal{L}\left(\mathbb{F}, \mathbb{F}^{\prime}\right)$ drops out of the category of Fréchet spaces and consequently it can not be considered as a projective limit of Banach spaces.

Considering the Fréchet space $\mathcal{H}\left(\mathbb{F}, \mathbb{F}^{\prime}\right)$ allows us to consider $\Psi_{\beta, g(x)}^{\infty} \circ$ $T^{\infty} g \circ \Phi_{\alpha, x}^{\infty}{ }^{-1}=\lim _{\longleftarrow} \Psi_{\beta, g(x)}^{k} \circ T^{k} g \circ \Phi_{\alpha, x}^{k}{ }^{-1}$ as a map with values in $\mathcal{H}\left(\mathbb{F}, \mathbb{F}^{\prime}\right)=$ $\lim _{\longleftarrow} \mathcal{H}^{k}\left(\mathbb{E}^{k}, \mathbb{E}^{\prime k}\right)$. Considering the auxiliary Banach spaces $\left\{\mathcal{H}^{k}\left(\mathbb{E}^{k}, \mathbb{E}^{\prime k}\right)\right\}_{k \in \mathbb{N}}$, the map $\lim _{\longleftarrow} \Psi_{\beta, g(x)}^{k} \circ T^{k} g \circ \Phi_{\alpha, x}^{k}{ }^{-1}$ is called a (generalized) smooth map since its factors are smooth [18, 19, 11].

Finally, following the argument of section 3 we get:

Theorem 4.2. The pair $\left(T^{\infty} g, g\right):\left(T^{\infty} M, \pi_{M}^{\infty}, M\right) \longrightarrow\left(T^{\infty} N, \pi_{N}^{\infty}, N\right)$ is a generalized vector bundle morphism. Moreover, the bundle morphism $\left(T^{\infty} g, g\right)$ is a vector bundle isomorphism if $g$ is a diffeomorphism.

Remark 4.3. It is easy to check that $\left(T^{\infty} g, T^{k} g\right):\left(T^{\infty} M, \pi_{M}^{\infty, k}, T^{k} M\right) \longrightarrow$ $\left(T^{\infty} N, \pi_{N}^{\infty, k}, T^{k} N\right), 1 \leq k<\infty$, is also a vector bundle morphism where $\pi_{M}^{\infty, k}$ maps the class $[\gamma, x]_{\infty}$ to $[\gamma, x]_{k}$. 


\section{Applications And examples}

In this section first, we settle our results to the special case of $f: M \longrightarrow$ $N$, where $f$ is an immersion and $N$ is a Riemannian Hilbert manifold. Then we study the vector bundle dependence on convex combination of connection maps and finally the case of manifold of $C^{r}$ maps between manifolds $M$ and $N$ is considered.

Example 5.1. Let $M$ and $N$ be two smooth manifolds modeled on the Hilbert spaces $\mathbb{E}$ and $\mathbb{F}$ respectively and $f: M \longrightarrow N$ be a smooth immersion. Moreover suppose that $h$ be a Riemannian metric on $N$ with the Levi-Civita connection $\nabla_{N}$. It is known that the immersion $f$ induces a Riemannian metric $g$ on $M$ defined by

$$
g(p)(u, v):=h(f(p))\left(d_{p} f u, d_{p} f v\right) ; \forall p \in M \text { and } \forall u, v \in T_{p} M .
$$

Denote by $\nabla_{M}$ the associated Levi-Civita connection of $g$. In what follows, we will show that $\nabla_{M}$ and $\nabla_{N}$ are $f$-related.

For $p \in M$ consider the charts $\left(U_{\alpha}, \phi_{\alpha}\right)$ and $\left(V_{\beta}, \psi_{\beta}\right)$ around $p$ and $f(p):=$ $q$ respectively. Setting

$$
g_{\alpha}\left(p_{0}\right):=g(p) \circ\left(d_{p_{0}} \phi_{\alpha}^{-1} \times d_{p_{0}} \phi_{\alpha}^{-1}\right): \mathbb{E} \times \mathbb{E} \longrightarrow \mathbb{R}
$$

and

$$
h_{\beta}\left(q_{0}\right):=h(q) \circ\left(d_{q_{0}} \psi_{\beta}^{-1} \times d_{q_{0}} \psi_{\beta}^{-1}\right): \mathbb{F} \times \mathbb{F} \longrightarrow \mathbb{R}
$$

we observe that

$$
\begin{aligned}
g_{\alpha}\left(p_{0}\right)(u, v) & =g(p)\left(d_{p_{0}} \phi_{\alpha}^{-1} u, d_{p_{0}} \phi_{\alpha}^{-1} v\right) \\
& =h(f(p))\left(d_{p_{0}} f \circ \phi_{\alpha}^{-1} u, d_{p_{0}} f \circ \phi_{\alpha}^{-1} v\right) \\
& =h_{\beta}\left(q_{0}\right)\left(d_{p_{0}} f_{\beta \alpha} u, d_{p_{0}} f_{\beta \alpha} v\right)
\end{aligned}
$$

where $p_{0}:=\phi(p), q_{0}:=f_{\beta \alpha}\left(p_{0}\right)$ and $f_{\beta \alpha}:=\psi_{\beta} \circ f \circ \phi_{\alpha}^{-1}$. As a consequence of (10) and Leibniz's rule we have

$$
\begin{aligned}
& d g_{\alpha}\left(p_{0}\right) \cdot w(u, v)=d h_{\beta}\left(q_{0}\right) \cdot d_{p_{0}} f_{\beta \alpha} w\left(d_{p_{0}} f_{\beta \alpha} u, d_{p_{0}} f_{\beta \alpha} v\right) \\
& +h_{\beta}\left(q_{0}\right)\left(d_{p_{0}}^{2} f_{\beta \alpha}(w, u), d_{p_{0}} f_{\beta \alpha} v\right)+h_{\beta}\left(q_{0}\right)\left(d_{p_{0}} f_{\beta \alpha} u, d_{p_{0}}^{2} f_{\beta \alpha}(w, v)\right)
\end{aligned}
$$

Now, using the Koszul formula (e.g. [9, 14]) we get

$$
\begin{gathered}
g_{\alpha}\left(p_{0}\right)\left(\Gamma_{\alpha}^{M}\left(p_{0}\right)(u, v), w\right)=\frac{1}{2}\left\{\begin{array}{c}
d g_{\alpha}\left(p_{0}\right) \cdot u(v, w)+d g_{\alpha}\left(p_{0}\right) \cdot v(u, w) \\
- \\
\left.-d g_{\alpha}\left(p_{0}\right) \cdot w(u, v)\right\} \\
=\frac{1}{2}\left\{d h_{\beta}\left(q_{0}\right) \cdot d_{p_{0}} f_{\beta \alpha} u\left(d_{p_{0}} f_{\beta \alpha} v, d_{p_{0}} f_{\beta \alpha} w\right)+h_{\beta}\left(q_{0}\right)\left(d_{p_{0}}^{2} f_{\beta \alpha}(u, v), d_{p_{0}} f_{\beta \alpha} w\right)\right. \\
+h_{\beta}\left(q_{0}\right)\left(d_{p_{0}} f_{\beta \alpha} v, d_{p_{0}}^{2} f_{\beta \alpha}(u, w)\right) \\
+d h_{\beta}\left(q_{0}\right) \cdot d_{p_{0}} f_{\beta \alpha} v\left(d_{p_{0}} f_{\beta \alpha} u, d_{p_{0}} f_{\beta \alpha} w\right)+h_{\beta}\left(q_{0}\right)\left(d_{p_{0}}^{2} f_{\beta \alpha}(v, u), d_{p_{0}} f_{\beta \alpha} w\right) \\
+h_{\beta}\left(q_{0}\right)\left(d_{p_{0}} f_{\beta \alpha} u, d_{p_{0}}^{2} f_{\beta \alpha}(v, w)\right) \\
-d h_{\beta}\left(q_{0}\right) \cdot d_{p_{0}} f_{\beta \alpha} w\left(d_{p_{0}} f_{\beta \alpha} u, d_{p_{0}} f_{\beta \alpha} v\right)-h_{\beta}\left(q_{0}\right)\left(d_{p_{0}}^{2} f_{\beta \alpha}(w, u), d_{p_{0}} f_{\beta \alpha} v\right) \\
\left.-h_{\beta}\left(q_{0}\right)\left(d_{p_{0}} f_{\beta \alpha} u, d_{p_{0}}^{2} f_{\beta \alpha}(w, v)\right)\right\}
\end{array}\right.
\end{gathered}
$$


$=h_{\beta}\left(q_{0}\right)\left(\Gamma_{\beta}^{N}\left(q_{0}\right)\left(d_{p_{0}} f_{\beta \alpha} u, d_{p_{0}} f_{\beta \alpha} v\right), d_{p_{0}} f_{\beta \alpha} w\right)+h_{\beta}\left(q_{0}\right)\left(d_{p_{0}}^{2} f_{\beta \alpha}(u, v), d_{p_{0}} f_{\beta \alpha} w\right)$

for any $u, v, w \in \mathbb{E}$. On the other hand equation (10) now reads

$$
g_{\alpha}\left(p_{0}\right)\left(\Gamma_{\alpha}^{M}\left(p_{0}\right)(u, v), w\right)=h_{\beta}\left(q_{0}\right)\left(d_{p_{0}} f_{\beta \alpha} \Gamma_{\alpha}^{M}\left(p_{0}\right)(u, v), d_{p_{0}} f_{\beta \alpha} w\right) .
$$

Since $g$ is non-degenerate we deduce that

$$
d_{p_{0}} f_{\beta \alpha} \Gamma_{\alpha}^{M}\left(p_{0}\right)(u, v)=\Gamma_{\beta}^{N}\left(q_{0}\right)\left(d_{p_{0}} f_{\beta \alpha} u, d_{p_{0}} f_{\beta \alpha} v\right)+d_{p_{0}}^{2} f_{\beta \alpha}(u, v) .
$$

However, the last equality is nothing but the local compatibility condition, described by remark 2.7, for the $f$-related connections $\nabla_{M}$ and $\nabla_{N}$.

As a consequence of theorems 2.11, 3.2 and 4.2 for $k \in \mathbb{N} \cup\{\infty\}, T^{k} M$ and $T^{k} N$ admit vector bundle structures and in this case $\left(T^{k} f, f\right):\left(T^{k} M, \pi_{M}^{k}, M\right)$ $\longrightarrow\left(T^{k} N, \pi_{N}^{k}, N\right)$ becomes a vector bundle morphism. Moreover if $f$ is an diffeomorphism (isometry) then, $T^{k} f$ is a vector bundle isomorphism. In this case, for $k \in \mathbb{N}$, with respect to the induced metrics introduced in section 3.3 of [26], $T^{k} g$ is also an isometry.

Example 5.2. Let $K=(\stackrel{1}{K}, \ldots, \stackrel{k}{K})$ and $\bar{K}=(\stackrel{1}{\bar{K}}, \ldots, \stackrel{k}{\bar{K}})$ be two connection maps on $T^{k} M$ and $0 \leq \lambda \leq 1$. We note that for any $1 \leq a \leq k-1$,

$$
\begin{aligned}
(\lambda K+(1-\lambda) \bar{K})^{k} \circ \mathbb{J}^{a} & =\lambda \stackrel{k}{K} \circ \mathbb{J}^{a}+(1-\lambda) \stackrel{k}{\bar{K}} \circ \mathbb{J}^{a} \\
& =\lambda \stackrel{k-a}{K}+(1-\lambda) \bar{K} \\
& =(\lambda K+(1-\lambda) \bar{K})^{k-a}
\end{aligned}
$$

and

$$
\begin{aligned}
(\lambda K+(1-\lambda) \bar{K})^{k} \circ \mathbb{J}^{k} & =\lambda \stackrel{k}{K} \circ \mathbb{J}^{k}+(1-\lambda) \stackrel{k}{K} \circ \mathbb{J}^{k} \\
& =\lambda \pi_{M *}^{k}+(1-\lambda) \pi_{M *}^{k}=\pi_{M *}^{k}
\end{aligned}
$$

that is the convex combination of $K$ and $\bar{K}$

$$
\lambda K+(1-\lambda) \bar{K}=(\lambda \stackrel{1}{K}+(1-\lambda) \stackrel{1}{K}, \ldots, \lambda \stackrel{k}{K}+(1-\lambda) \stackrel{k}{K})
$$

is a connection map too. Moreover it is easily seen that for any $u=$ $\left(x, \xi_{1}, \ldots, \xi_{k}\right)$ and $\left(u, y, \eta_{1}, \ldots, \eta_{k}\right) \in T_{u} T^{k} M$

$$
\begin{aligned}
& \left.(\lambda K+(1-\lambda) \bar{K})\right|_{U_{\alpha}}\left(u ; y, \eta_{1}, \ldots, \eta_{k}\right)=\bigoplus_{i=1}^{k}\left(x, \eta_{i}+\right. \\
& \left.\left(\lambda \stackrel{1}{M_{\alpha}}(u)+(1-\lambda) \stackrel{1}{M_{\alpha}}(u)\right) \eta_{i-1}+\cdots+\left(\lambda \stackrel{i}{M_{\alpha}}(u)+(1-\lambda) \stackrel{i}{M_{\alpha}}(u)\right) y\right) .
\end{aligned}
$$

As a consequence of theorem 2.11, for any $x \in M$

$$
\begin{aligned}
\left(T^{k} M_{\lambda K+(1-\lambda) \bar{K}}\right)_{x} & =\left(T^{k} M_{\lambda K}\right)_{x}+\left(T^{k} M_{(1-\lambda) \bar{K}}\right)_{x} \\
& =\lambda\left(T^{k} M_{K}\right)_{x}+(1-\lambda)\left(T^{k} M_{\bar{K}}\right)_{x}
\end{aligned}
$$

where $T^{k} M_{K}, T^{k} M_{\bar{K}}$ and $T^{k} M_{\lambda K+(1-\lambda) \bar{K}}$ denote the vector bundle structures on $\pi_{M}^{k}: T^{k} M \longrightarrow M$ induced by the connection maps $K, \bar{K}$ and $\lambda K+(1-\lambda) \bar{K}$ respectively. 
This last result means that the fibres of the vector bundle structure induced by the convex combination $\lambda K+(1-\lambda) \bar{K}$, is the convex combination of fibres of the vector bundle structures induced by $K$ and $\bar{K}$.

A similar argument holds for any convex combination $\sum_{i=1}^{n} \lambda_{i} K_{i}$ of connection maps.

Example 5.3. Let $N$ be a $C^{\infty}$ compact manifold and $M$ be a $C^{\infty}$ Banach (possibly infinite dimensional) manifold with a linear connection $\nabla_{M}$. According to $8 C^{r}(N, M)$, the space of all $C^{r}$ maps $0 \leq r<\infty$ from $N$ to $M$, forms a Banach manifold with the following charts. Let exp : $\mathcal{O} \subset$ $T M \longrightarrow M$ be the exponential map corresponding to the linear connection $\nabla_{M}$. Moreover suppose that $\mathcal{D}$ be an open neighborhood of the zero section in $T M$ such that $\left.\left(\pi_{M}^{1}, \exp \right)\right|_{\mathcal{D}}$ form $\mathcal{D}$ to $\left(\pi_{M}^{1}, \exp \right)(\mathcal{D}) \subset M \times M$ is a diffeomorphism. For the $C^{r}$ map $h: N \longrightarrow M$ the chart $\left(\phi_{h}, U_{h}\right)$ defined by

$$
C^{r}(\exp ): C^{r}\left(h^{*} \mathcal{D}\right) \longrightarrow C^{r}(N, M) ; \xi \longmapsto \exp \circ \xi .
$$

In the notation above $C^{r}\left(h^{*} \mathcal{D}\right)$ is the set of all sections $\xi: N \longrightarrow T M$ with the property $\pi_{M}^{1} \circ \xi=h$. In this case $C^{r}\left(h^{*} \mathcal{D}\right)$ becomes a Banach space with the norm

$$
\|\xi\|_{C^{r}}=\sum_{j=0}^{r}\left\|\nabla^{j} \xi\right\|_{C^{0}}:=\sum_{j=0}^{r} \sup \left\|\nabla^{j} \xi(p)\right\|_{p \in N} .
$$

which serves as the model space of $C^{r}(N, M)$.

Moreover the connection $\nabla_{M}$ induces a connection on $C^{r}(N, M)$ with the connection map $C^{r}\left(\nabla_{M}\right): T T C^{r}(N, M) \simeq C^{r}(N, T T M) \longrightarrow C^{r}(N, T M) \simeq$ $T C^{r}(N, M)$ which maps $A \in C^{r}(N, T T M)$ to $C^{r}\left(\nabla_{M}\right)(A)=\nabla_{M} \circ A$. Since $\nabla_{M}$ is a linear connection, so $C^{r}\left(\nabla_{M}\right)$ also is a linear connection ([8] Theorem 5.4).

According to theorem 2.11, $T^{k} M$ and $T^{k} C^{r}(N, M)$ admits vector bundle structures on $M$ and $C^{r}(N, M)$ respectively. Moreover $T^{k} C^{r}(N, M) \simeq$ $\oplus_{j=1}^{k} T C^{r}(N, M)$ and $C^{r}\left(N, T^{k} M\right) \simeq C^{r}\left(N, \oplus_{j=1}^{k} T M\right)$ are isomorphic vector bundles over $C^{r}(N, M)$.

For the Banach manifolds $M$ and $M^{\prime}$ and the smooth map $g: M \longrightarrow$ $M^{\prime}$ the map $C^{r}(g): C^{r}(N, M) \longrightarrow C^{r}\left(N, M^{\prime}\right)$ defined by $f \longmapsto g \circ f$ is differentiable and $T C^{r}(g)=C^{r}(T g)[$ ] .

Now, suppose that $\nabla_{M}$ and $\nabla_{M^{\prime}}$ be two $g$-related connections on $M$ and $M^{\prime}$ respectively. Then

$$
\begin{aligned}
C^{r}\left(\nabla_{M^{\prime}}\right) \circ T T C^{r}(g) & =C^{r}\left(\nabla_{M^{\prime}}\right) \circ C^{r}(T T g)=C^{r}\left(\nabla_{M^{\prime}} \circ T T g\right) \\
& =C^{r}\left(T g \circ \nabla_{M}\right)=T C^{r}(g) \circ C^{r}\left(\nabla_{M}\right)
\end{aligned}
$$

that is $C^{r}\left(\nabla_{M}\right)$ and $C^{r}\left(\nabla_{M^{\prime}}\right)$ are $C^{r}(g)$-related. As a consequence of theorem 3.2, $T^{k} g: T^{k} M \longrightarrow T^{k} M^{\prime}$ and $T^{k} C^{r}(g): T^{k} C^{r}(N, M) \longrightarrow$ $T^{k} C^{r}\left(N, M^{\prime}\right)$ are vector bundle morphisms.

\section{APPENDIX}

In this section, using the chain rule formula (1) we prove lemma 3.1. For $\left(x, \xi_{1}, \ldots, \xi_{k}\right) \in U_{\alpha} \times \mathbb{E}^{k}$, define the curve $\bar{\mu}_{k}$ inductively as in section 3 by 
$\bar{\mu}_{1}(t)=x+t \xi_{1}, \bar{\mu}_{2}(t)=\bar{\mu}_{1}(t)+\frac{t^{2}}{2}\left\{2 \xi_{2}-\stackrel{1}{M} \alpha\left(x, \xi_{1}\right) \xi_{1}\right\}$ and for $i \geq 2$,

$$
\begin{gathered}
\bar{\mu}_{i}(t)=\bar{\mu}_{i-1}(t)+\frac{t^{i}}{i}\left\{i \xi_{i}-M_{\alpha}\left(x, \xi_{1}\right) \frac{\bar{\mu}_{i-1}^{(i-1)}(0)}{(i-2) !}-\ldots\right. \\
\left.\stackrel{i-1}{\alpha}_{\alpha}\left(x, \xi_{1}, \frac{1}{2 !} \bar{\mu}_{i-1}^{(2)}(0), \ldots, \frac{1}{(i-1) !} \bar{\mu}_{i-1}^{(i-1)}(0)\right) \xi_{1}\right\} .
\end{gathered}
$$

Lemma 6.1. Let $\bar{\mu}:=\bar{\mu}_{k}$ be the map defined above, $\mathcal{O} \subseteq \mathbb{E}$ be open and $f: \mathcal{O} \longrightarrow \mathbb{E}$ be any smooth map. Then

$$
\left.\frac{\partial^{k}}{\partial s \partial t^{k-1}}\left(f \circ \bar{d}_{k}\right)(t, s)\right|_{t=s=0}=\left.(f \circ \bar{\mu})^{(k)}(t)\right|_{t=0}
$$

where

$$
\bar{d}_{k}:(-\epsilon, \epsilon)^{2} \longrightarrow \mathbb{E} ;(t, s) \longmapsto \sum_{i=1}^{k-1} \frac{t^{i}}{i !}\left(\bar{\mu}^{(i)}(0)+s \bar{\mu}^{(i+1)}(0)\right) .
$$

Proof. Using the chain rule formula (1) we observe that

$$
\begin{aligned}
& \left.\frac{\partial^{k}}{\partial s \partial t^{k-1}}\left(f \circ \bar{d}_{k}\right)(t, s)\right|_{t=s=0} \\
= & \left.\frac{\partial}{\partial s}\right|_{s=0}\left\{d f\left(x+s \xi_{1}\right)\left[\bar{\mu}^{(k-1)}(0)+s \bar{\mu}^{(k)}(0)\right]\right. \\
& +\sum_{l_{1}+l_{2}=k-1} a_{l_{1}, l_{2}}^{k-1} d^{2} f\left(x+s \xi_{1}\right)\left[\bar{\mu}^{\left(l_{1}\right)}(0)+s \bar{\mu}^{\left(l_{1}+1\right)}(0), \bar{\mu}^{\left(l_{2}\right)}(0)+s \bar{\mu}^{\left(l_{2}+1\right)}(0)\right]+ \\
& \left.\cdots+d^{k-1}\left(x+s \xi_{1}\right)\left[\bar{\mu}^{(1)}(0)+s \bar{\mu}^{(2)}(0), \ldots, \bar{\mu}^{(1)}(0)+s \bar{\mu}^{(2)}(0)\right]\right\} \\
= & d f(x)\left[\bar{\mu}^{(k)}(0)\right]+d^{2} f(x)\left[\bar{\mu}^{(1)}(0), \bar{\mu}^{(k-1)}(0)\right]+(k-1)\left\{d ^ { 2 } f ( x ) \left[\bar{\mu}^{(2)}(0),\right.\right. \\
& \left.\bar{\mu}^{(k-2)}(0)\right]+d^{2} f(x)\left[\bar{\mu}^{(1)}(0), \bar{\mu}^{(k-1)}(0)\right]+d^{3} f(x)\left[\bar{\mu}^{(1)}(0), \bar{\mu}^{(1)}(0),\right. \\
& \left.\left.\bar{\mu}^{(k-2)}(0)\right]\right\}+\cdots+(k-1) d^{k-1} f(x)\left[\bar{\mu}^{(1)}(0), \ldots, \bar{\mu}^{(1)}(0), \bar{\mu}^{(2)}(0)\right] \\
& +d^{k} f(x)\left[\bar{\mu}^{(1)}(0), \ldots, \bar{\mu}^{(1)}(0)\right] \\
= & d f(x)\left[\bar{\mu}^{(k)}(0)\right]+k d^{2} f(x)\left[\bar{\mu}^{(1)}(0), \bar{\mu}^{(k-1)}(0)\right]+\frac{k(k-1)}{2} d^{2} f(x)\left[\bar{\mu}^{(2)}(0),\right. \\
& \left.\bar{\mu}^{(k-2)}(0)\right]+\cdots+d^{k} f(x)\left[\bar{\mu}^{(1)}(0), \ldots, \bar{\mu}^{(1)}(0)\right] \\
= & (f \circ \bar{\mu})^{(k)}(0)
\end{aligned}
$$

as we claimed.

Finally, we leave it to the reader to verify that

Lemma 6.2. For any differentiable function $f: O \subseteq \mathbb{E} \longrightarrow \mathbb{E}$ and the maps $\bar{c}_{1}(t, s, h):=x+h \xi_{1}+s y+t \xi_{1}+\cdots+t^{k} \xi_{k}, \bar{\gamma}_{1}(t, h):=\bar{c}_{1}(t, 0, h), \ldots$, $\bar{c}_{i}(t, s, h):=x+s y+t \xi_{1}+\cdots+t^{i-2} \xi_{i-2}+t^{i-1}\left(\xi_{i-1}+h i \xi_{i}\right)+t^{i} \xi_{i}+\cdots+t^{k} \xi_{k}$, $\bar{\gamma}_{i}(t, h):=\bar{c}_{i}(t, 0, h), 1 \leq i \leq k$, and $\bar{c}(t, s)=x+s y+t \xi_{1}+\cdots+t^{k} \xi_{k}$ the following properties hold.

$i$.

$$
\left.\frac{\partial^{j+1}}{\partial h \partial s \partial t^{j-1}} \sum_{i=1}^{k}\left(f \circ \bar{c}_{i}\right)(t, s, h)\right|_{t=s=h=0}=\left.\frac{\partial^{j+1}}{\partial s \partial t^{j}}(f \circ \bar{c})(t, s)\right|_{t=s=0}
$$


ii.

$$
\left.\frac{\partial^{j}}{\partial h \partial t^{j-1}} \sum_{i=1}^{k}\left(f \circ \bar{c}_{i}\right)(t, s, h)\right|_{t=s=h=0}=\left.\frac{\partial^{j}}{\partial t^{j}}(f \circ \bar{c})(t, s)\right|_{t=s=0}
$$

$j=1, \ldots, k$.

\section{REFERENCES}

[1] M. Aghasi and A. Suri, Splitting theorems for the double tangent bundles of Fréchet manifolds. Balkan journal of geometry and its applications, No. 15, Vol.2, (2010), pp. $1-13$.

[2] V. I. Averbuh and O. G. Smolyanov, Differentiation theory in linear topological spaces, Uspehi Mat. Nauk 6, Russian Math. Surveys, No.6, (1967), pp. 201-258.

[3] V. I. Averbuh and O. G. Smolyanov, The various definitions of the derivative in linear topological spaces, Russian Math. Surveys, No. 23, (1968), pp. 67-113.

[4] I. Bucataru, Linear connections for systems of higher order differential equations, Houston J. Math., Vol.31, No.(2),(2005) pp. 315-332.

[5] M. De León and P. Rodriguez, Generalised Classical Mechanics and Field Theory, Amsterdam, North-Holland, 1985.

[6] C.T.J. Dodson and G.N. Galanis, Second order tangent bundles of infinite dimensional manifolds, J. Geom. Phys., 52 (2004), pp. 127-136.

[7] C.T.J. Dodson, G.N. Galanis and E. Vassiliou, Isomorphism classes for Banach vector bundle structures of second tangents, Math. Proc. Camb. Phil. Soc., 141 (2006), pp. 489-496.

[8] H. I. Eliasson, Geometry of manifolds of maps, J. Differentail Geometry, 1 (1967), pp. 169-194.

[9] P. Flaschel and W. Klingenberg, Riemannsche Hilbertmannigfaltigkeiten. Periodische Geodätische, Lecture Notes in Mathematics, No. 282, 1972.

[10] G.N. Galanis, Projective limits of Banach Lie groups, , Period. Math. Hungar., 32 (3), (1996), pp. 179-191.

[11] G.N. Galanis, Projective limits of Banach vector bundles, , Portugaliae Mathematica, vol. 55 Fasc. 1, (1998), pp. 11-24.

[12] R.S. Hamilton, The inverse function theorem of Nash and Moser, Bull. of Amer. Math. Soc., Vol.7, No.1 (1982), pp. 65-222.

[13] H.H. Keller, Differential calculus in locally convex spaces, Lecture notes in mathematics, No. 417, Springer-Verlag, 1974.

[14] W. Klingenberg, Riemannian geometry, Walter de Gruyter, Berlin, New york, 1995.

[15] O. V. Kunakovskaya, On smooth partition of unity over Banach manifolds, Russian mathematics (Iz. VUZ), (1997) Vol.41, No.10, pp.51-58.

[16] S. Lang, Fundumentals of differential geometry, Graduate Texts in Mathematics, vol. 191, Springer-Verlag, New York, 1999.

[17] J. W. Lloyd, Higher order derivatives in topological linear spaces, J. Austral. Math. Soc. 25 (Series A) (1978) pp. 348-361.

[18] J.A. Leslie, On a differential structure for the group of diffeomorphisms, Topology, 6 (1967), pp. 263-271.

[19] J.A. Leslie, Some Frobenious theorems in Global Analysis, J. Diff. Geom., 2 (1968), pp. 279-297.

[20] R. Miron, The geometry of higher order Lagrange spaces applications to Mechanics and Physics, Kluwer Academic publishers, 1997.

[21] A. Morimoto, Liftings of tensor fields and connections to tangent bundles of higher order, Nagoya Math. J., Vol. 40, (1970), pp. 99-120.

[22] H. H. Schaefer, Topological Vector Spaces, Springer-Verlag, Berlin, 1980.

[23] L. Schwartz, Cours d 'analyse, Hermann, Paris, 1967. 
[24] A. Suri and M. Aghasi, Connections and second order differential equations on infinite dimensional manifolds, Int. Electron. J. Geom. , Vol. 6, No. 2, , (2013), pp. $45-56$.

[25] A. Suri, Geometry of the double tangent bundles of Banach manifodls, J. Geom. Phys., Vol. 74, (2013), pp. 91-100.

[26] A. Suri, Higher order tangent bundles, http://arxiv.org/abs/1403.3111v1.

[27] A. Suri, Higher order frame bundles, preprint.

[28] E. Vassiliou, Transformations of Linear Connections, Period. Math. Hungar., 13 (1982), pp. 289-308.

[29] J. Vilms, Connections on tangent bundles, J. Diff. Geom. Vol.1, (1967) pp. 235-243.

[30] K. Yano and Sh. Ishihara, Differential geometry of tangent bundles of order 2, Kodai Math. Sem. Rep., Vol.20, (1968) pp. 318-354.

Department of Mathematics, Faculty of sciences, Bu-Ali Sina University, HAMEDAN 65178, IRAN.

E-mail address: ali.suri@gmail.com \& a.suri@math.iut.ac.ir \& a.suri@basu.ac.ir 